\title{
Modelling the chemo-mechanical responses of solid solutions far from equilibrium under heterogeneous stresses
}

\author{
Santiago P Clavijo ${ }^{1}$, Luis Espath ${ }^{2}$, Victor M Calo ${ }^{1,3,4}$ \\ ${ }^{1}$ School of Earth and Planetary Sciences, Curtin University, Kent Street, Bentley, Perth, WA 6102, \\ Australia \\ ${ }^{2}$ Department of Mathematics, RWTH Aachen University, Kackertstraße 9 C363, Aachen 52072, Germany \\ ${ }^{3}$ Curtin Institute for Computation, Curtin University, Kent Street, Bentley, Perth, WA 6102, Australia \\ ${ }^{4}$ Mineral Resources, Commonwealth Scientific and Industrial Research Organisation (CSIRO), \\ Kensington, Perth, WA 6152, Australia
}

\section{Key Points:}

- Mineral solid solutions

- Coupled reactive chemo-mechanical behaviour

- Thermodynamic pressure 


\begin{abstract}
We present a coupled thermodynamically-consistent framework for the reactive chemomechanical responses of solid solutions. Specifically, we focus on chemically active solid solutions subject to mechanical effects due to heterogeneous stresses distributions, where the stress generation process (pressure) is driven solely by volume changes associated with the chemical processes. Throughout this paper, we use the model to describe common geological processes. Furthermore, simulation results of a three-phases solid solution provide insights into the phenomena and verify the interleaving between the physical and chemical interactions at solid-state. In particular, we show the evolution of the thermodynamic pressure as the system goes to the steady state.
\end{abstract}

\title{
1 Introduction
}

In discussing systems undergoing volume changes, Truesdell (1984) $\S 5 \mathrm{C}-$ in the appendix A Theory of Multiphase Mixtures by Passman, Nunziato \& Walsh -identified the thermodynamical pressure as the conjugate power expenditure to this thermo-kinematic process, that is, the change of volume versus the thermodynamic pressure. The spherical part of the Cauchy tensor only provides the 'mechanical' contribution albeit essential to the thermodynamic pressure. Therefore, the spherical part of the Cauchy tensor does not completely describe the pressure. The thermodynamic pressure is defined as the negative variation of the Helmholtz free-energy with respect to the volumetric variations, that is, $p^{t h}=-\partial \Psi / \partial v$. In general at steady state, this thermodynamic pressure may be spatially inhomogeneous which implies that the system reaches equilibrium under non-hydrostatic stresses (Larché \& Cahn, 1973, 1978a, 1984, 1978b).

To model the physical and chemical responses that lead to inhomogeneous pressures, we propose a thermodynamically-consistent model for the description of the chemomechanical interactions of solid solutions far from equilibrium. Inhomogeneous stress states and therefore spatially inhomogeneous pressure distributions arise from either external loading induced by deformation across the solid boundaries or local volume changes associated with the evolution of chemical processes. In this work, we model the inhomogeneous pressure distributions that result from local volume changes. We show that nucleation and growth of new phases induce volumetric stresses that lead to spatially inhomogeneous pressure distributions. We use a constitutive relation for the elastic energy that relates the stress-assisted volume changes and stresses resulting from mechanical loading. Moreover, we incorporate an interfacial energy in the definition of the chemical energy. Using our thermodynamically-consistent framework, we model an elastic solid composed of three phases. As the phases diffuse and react, the solid undergoes volumetric stresses which drive the generation of the inhomogeneous pressure distribution. As mentioned above, we calculate this pressure as the negative of the variation of the Helmholtz free energy with respect to the specific volume. The material parameters in the simulation are in the range of common physical and chemical processes in geosciences. We model the elastic properties as a function of the volume fraction, as usually done in the theory of mixtures. However, to analyse the simplest scenario, we keep the elastic properties constant throughout the simulations. Thus, we focus our attention to the heterogeneities in the thermodynamical pressure.

An open research topic in geosciences is to characterise the sources that engender heterogeneous pressure distribution in metamorphic minerals, and most importantly, the pressure that defines the equilibrium conditions of the metamorphic system. Studies on metamorphic petrology and microstructural observations suggest the influence of mechanical effects upon chemically active metamorphic minerals (Milke et al., 2009; Tajčmanová et al., 2015; Hobbs \& Ord, 2016; Moulas et al., 2013; Vrijmoed \& Podladchikov, 2015; Wheeler, 2014; Zhong et al., 2017). Tajčmanová et al. (2015) study the effects of an inhomogeneous pressure distribution which can be maintained even in the microscale at 
ambient conditions. The study of minerals under residual pressure can be carried out by advanced observational techniques. Howell et al. (2010) use an analytical model that relates geometric features of both the host rock and the inclusion together with a quantitative birefringence analysis to study the residual stress of graphite inclusion in diamond. Their measurements show the distribution of internal inhomogeneous pressure around the graphite inclusion caused by the residual stresses. Thereby, we believe that the initial step for geoscientists must be the definition of all possible mechanisms that change the volume of the system which in turn impact the pressure. As a part of this effort, we detail the relation between the chemo-mechanical behaviour of metamorphic minerals and common physical and chemical processes in solid solutions. Our framework cannot completely describe a metamorphic rock as these systems are composed of several minerals together with grain-boundaries whose crystalline structure, chemical, and mechanical properties differ for each mineral. Besides, during metamorphism, these rocks interact with fluids which strongly define the grade of metamorphism that eventually alters the properties of the rock. Nevertheless, the aforementioned model sets the basis for a thermodynamic treatment of the heterogeneous pressure distributions in metamorphic minerals such as sillimanite, kyanite, staurolite, andalusite, and garnets as they can be modelled as solid solutions (one or more phases).

The remainder of the paper has the following structure. Section 2 introduces the thermodynamical basis of the model. Section 2.2 covers the definition of the network model proposed by Larché and Cahn as well as the nature of solidity while allowing for compositional changes. Section 2.3 describes the elastic energy, in particular, the coupling between the chemical and the mechanical processes. Section 2.4 outlines the definition of a chemical energy potential which relates interfacial interactions between the phases. We also describe the Ostwald ripening effect and phase separation processes. Section 2.5 embraces the balance equations that rule the evolution of an elastic solid undergoing chemical processes. In this section, we also show how to estimate the thermodynamic pressure of the system from the Helmholtz free energy. Finally, in section 3, we study the evolution of a solid composed of three phases where one of the phases results from a forward chemical reaction. As the new phase grows and nucleate, the solid undergoes elastic stresses which drive the generation of the inhomogeneous pressure distributions.

\section{A thermodynamically-consistent description of chemo-mechanical interactions in solid solutions}

In this section, we describe how the interactions between elasticity, diffusion, and chemical reactions engender inhomogeneous pressure distributions in a solid solution.

The Helmholtz free energy functional accounts for the contributions from the mechanical and chemical responses of the system. With regards to the chemical energy, we use a potential that characterises the dynamics of a solid system which may undergo phase separation in solid state. The interfacial interactions between the phases drives the phase separation process. Analogously, we describe the solid as a compressible neo-Hookean elastic material. We treat the solid as a continuum body subject to a motion described by a deformation field. The kinematics of the motion of the particles in the body define the deformation field. The solid is composed by several phases. In the continuum mechanics literature, such systems are commonly called solid-species solutions. Henceforth, we adopt this denomination.

We derive a set of balance equations in the form of partial differential equations which define how the mass, linear and angular momenta, internal energy and entropy of the system vary in time as the deformation and chemical processes take place. As suggested in Gurtin et al. (2010); Dal \& Miehe (2015); Miehe et al. (2016); Tsagrakis \& Aifantis (2017), three primary fields govern the coupled chemo-mechanical responses of a solidspecies solution: the deformation field, the species concentrations, and the chemical po- 
tentials. Our description of a solid-species solution builds on the Larché-Cahn model of solids (Larché \& Cahn, 1973, 1978a, 1984, 1978b). This model defines the relative chemical potential as a result of the Larché-Cahn derivative (Gurtin et al., 2010; Larché \& Cahn, 1973). The relative chemical potential accounts for the fact that two different species may share the same lattice site in the crystalline structure of the solid. The relative chemical potential characterizes the energy exchange caused by species transport and transformation. This chemical potential describes how the energy changes when one species increases its concentration while simultaneously reducing another one while keeping all other species concentrations fixed. The relative chemical potential expresses the constraints imposed on the diffusion processes by the lattice of the crystalline structure. That is, the diffusion process is only feasible if the variation of the local composition of one species induces a complementary change in another species concentration.

Our formulation involves non-Fickian diffusion processes as it relates interfacial diffusion. These interfacial interactions are important since they can describe, for example, spontaneous phase separation processes as well as the Ostwald ripening and GibbsThomson effects.

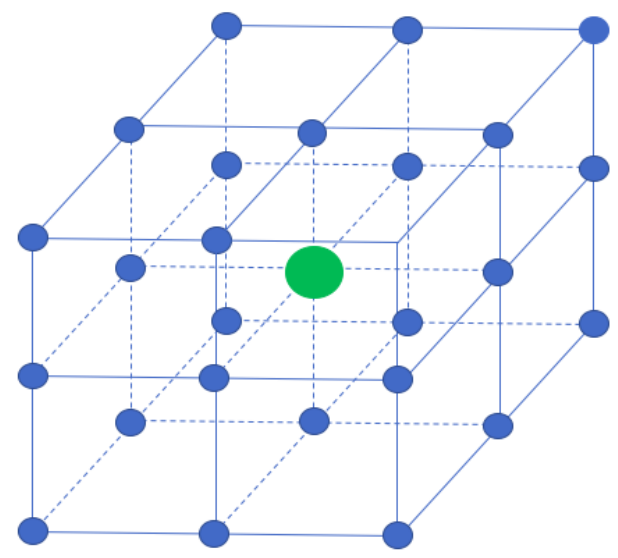

Figure 1 shows an idealized cubic crystalline structure. The atoms inside the crystalline structure are more energetically stable than the surface ones since they are bounded by more neighboring atoms.

\subsection{Interfacial interactions}

The interfacial interactions explain the Ostwald ripening effect which is common during the textural evolution of metamorphic rocks (Miyazaki, 1996, 1991; Eberl et al., 1990; Nemchin et al., 2001). This ripening effect is a thermodynamically-driven spontaneous process in spatially heterogeneous solutions, with small and large aggregates, as the thermodynamic system moves to a lower energy state by minimizing the interface length. The small aggregates in the solid solution dissolve into the solution and reprecipitate onto the surface of the larger aggregates since the smaller aggregates are less energetically favoured. To illustrate the latter description and without loss of generality, let us consider a solid solution with a cubic crystalline structure as depicted in Figure 1 . The green atom is the most energetically stable atom in the crystalline structure due to its six neighboring atoms. Meanwhile, the blue atoms on the surface are less energetically stable since they are only bounded by five or fewer neighboring atoms. The aggregates with more green (interior) atoms are energetically favoured and therefore, more stable. Thus, the most stable structures in the solution are the larger aggregates as they contain more energetically stable atoms. Consequently, as the system tries to minimize 
its free energy, the less stable structures, namely the smaller aggregates, tend to dissolve into the solution and precipitate on the surface of the most stable structures. This mechanism shrinks the smaller aggregates and grows the larger ones, a process that increases the overall aggregate size on average in solution. For instance, Figure 2 (a) shows an idealised representation of a rock composed of two aggregates and matrix where the small aggregate of the red phase is subject to Ostwald ripening. Eventually, the smaller red aggregate completely dissolves and precipitates leading to the growth of the larger aggregate. Figure 2 (b)-(c) portrait the intermediate and final stages of such process, respectively.

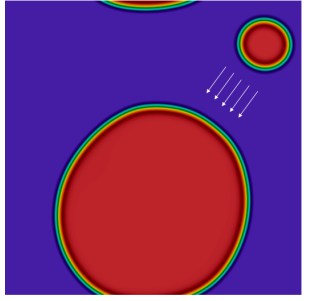

(a)

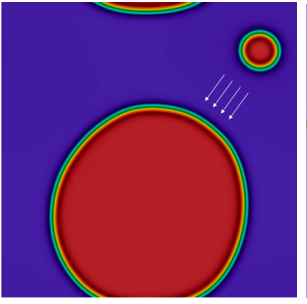

(b)

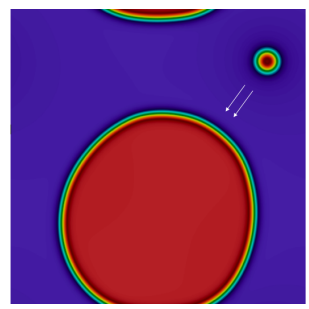

(d)

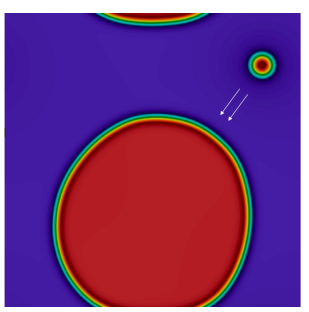

(c)

Figure 2 (a) depicts an idealised configuration of a solid composed of two aggregates and matrix. The dynamics described by the Ostwald ripening effect control the dissolution of the small aggregate and eventually its precipitation onto the surface of the larger aggregate once the solution supersaturates. Moreover (b), (c) and (d) sketch this evolution mechanism. As the concentration of the smaller inclusion is depleted, the stress field changes and such process drives the final shape of the large inclusion.

\subsection{Crystalline structure and mass constraint}

To understand the impact of the mechanical and chemical processes on solids requires the description of the nature of solidity and its properties. Gibbs pioneering work introduced a theory for the equilibrium thermodynamics of solids under non-hydrostatic conditions where dissolution and accretion at the solid-fluid interfaces is possible (Gibbs, 1878). As a particular example, Gibbs's model describes a non-hydrostatic stress distribution at the contact point of a solid with more than one fluid. This isotropic stress (commonly denoted pressure) induces a difference in the fluid pressure, $p^{\text {fluid }}$, at the solidfluid interface. The latter implies that the chemical potential of the dissolved solid in each fluid is also different. Nonetheless, the Gibbs's theory does not quantify the internal adjustment in the solid lattice caused by the compositional changes as the concept of solid state diffusion did not exist (Gibbs, 1878; Sekerka \& Cahn, 2004; Larché \& Cahn, 1978a). We now model elastic solids that allow for compositional changes while remaining in the solid state. Consequently, we adopt the network model proposed by Larché \& Cahn (1978a). This model relies on the idea of a network embedded in the structure of the solid which allows for the definition of a displacement field, and therefore a strain of the solid (Miehe et al., 2016; Gurtin et al., 2010; Larché \& Cahn, 1973). As a result, 


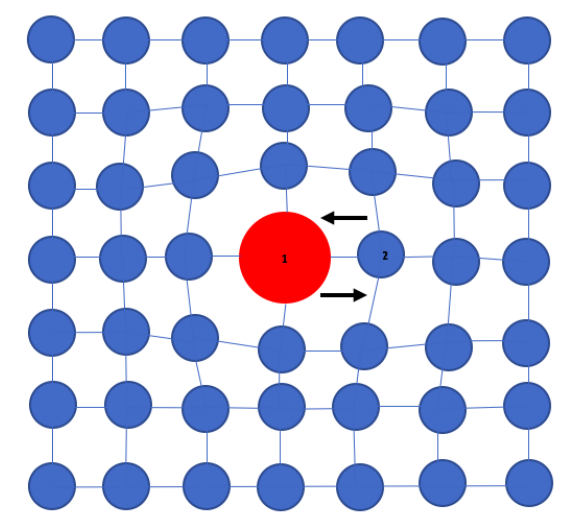

Figure 3 depicts the Larché and Cahn representation of the network embedded in the crystalline structure of a solid. The solid is composed of two species sketched as red and blue circles. The figure shows a coherent transition between the phases together with the stress-assisted volume changes mechanism. The transport of the red atom from the lattice site 1 to the lattice site 2 induces volumetric stresses.

the strain quantifies the deformation of the network with respect to a reference network configuration commonly set as an undeformed state. In several natural and engineering materials, such as, minerals, polymers, and metals, a solid network can be identified. For instance, the unit cell of the crystalline structure of minerals, which arranges the atoms in a systematic and repeating pattern, acts as a network. We restrict our attention to saturated systems, such that

$$
\sum_{\alpha=1}^{N} \varphi^{\alpha}=1
$$

where the order parameter $\varphi_{\alpha}$ accounts for the dimensionless concentration of the $\alpha$-th species. When the solid is solely composed of the diffusing species the mass constraint given by (2.2) must hold. Figure 3 depicts the crystalline structure of a solid composed of two different species (drawn as the red and blue circles), and it corresponds to the case where adjacent phases have coherent transitions, namely the orientation of their crystalline structure coincides. When a new species grows and nucleates the solid network must accounts for the lattice misalignment between the phases. According to Larché \& Cahn $(1973,1978 \mathrm{a}, 1984,1978 \mathrm{~b})$, the growth and nucleation of new phases require describing non-coherent phase transitions by defining a crystalline structure and proper orientations of the mechanical properties for each phase. In our framework, the mass transport and the nucleation and growth of new species induced by chemical reactions generate elastic strains. In Figure 3, for instance, the transport of the red atom from the lattice site (1) to (2) must contribute to distort the crystalline structure and therefore, to generate elastic strains. Henceforth, we denote such mechanism as stress-assisted volume changes. The transport of the red atom from the lattice site (1) to (2) requires the movement of other atoms towards the lattice site (1) since the mass constraint given by (2.2) must always hold. Thus, we restrict our attention to the case where mass transport by vacancies is not feasible. In multicomponent systems, we also identify the partial pressure $p_{\alpha}=\varphi_{\alpha} p$ as the pressure related to the $\alpha$-th species, with a concentration $\varphi_{\alpha}$. 


\subsection{Elastic energy}

The elastic energy of a solid defines the potential energy stored in the solid material as work is performed to change its volume or distort its shape. External forces applied through the solid boundaries, body forces due to gravity, electric and magnetic fields, thermal swelling/shrinkage, and internal adjustment caused by compositional changes transfer elastic energy to the solid.

The minerals that compose rocks accommodate these processes along their evolution. Shear zones and overburden are typical examples of external loading applied to a rock. The chemical interactions caused by diffusion and reaction, where atoms arrange to form a material with a crystal structure, are examples of internal adjustment caused by compositional changes. Exhumation of deep crustal metamorphic rocks involves thermal swelling and shrinkage due to the temperature gradient in the crust.

All elastic responses allow the solid to recover its original configuration when the external source is removed. The solid recovers its shape and volume. In our framework, the variations in local species concentration are scaled by a swelling parameter $\omega$ which measures the impact of a change in local species concentration on the generation of volumetric stresses. The parameter $\omega$ is related to the crystalline structure of the solid and its mechanical properties.

Figure 4 depicts the elastic energy $\hat{\psi}^{e l}$ as a function of the local species concentration parametrised by the swelling parameter $\omega$ for a two phases system. With fixed boundaries, the stress variations are only due to the changes of the species concentration. As we can see in Figure 4, the elastic energy increases as the swelling parameter becomes larger. Therefore, as long as the local species concentrations change with respect to the initial distribution, the solid undergoes elastic deformation. The interaction between diffusion and deformation change the rates of both processes.

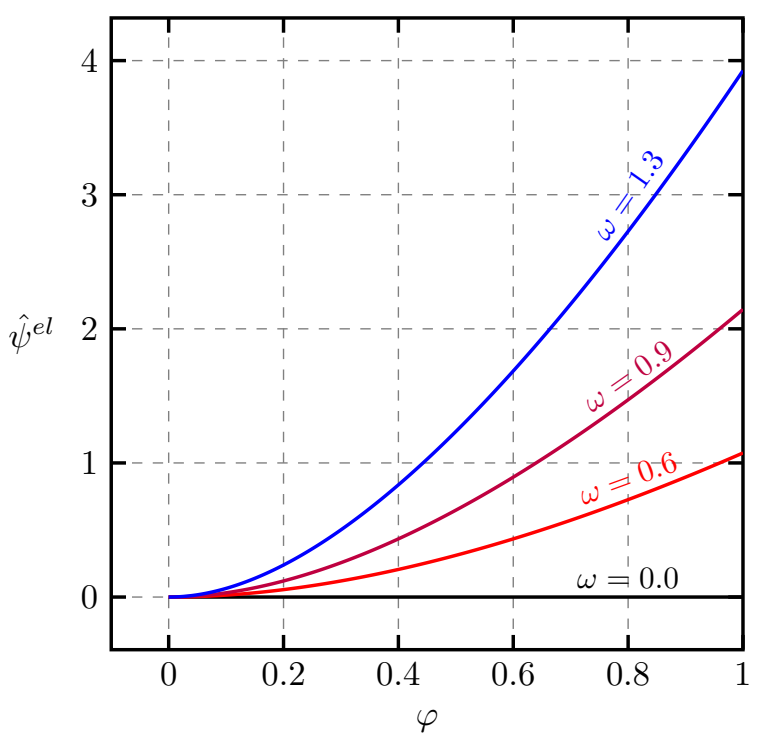

Figure 4 shows the elastic energy $\hat{\psi}^{e l}$ as a function of the local concentration. The parametrisation shows the effect of the swelling parameter $\omega$ on the elastic energy $\hat{\psi}^{e}$. 


\subsection{Chemical energy}

Solid solutions are complex systems composed of several phases. The phase interfaces may be of non-zero thickness where the physical and chemical properties vary from one phase to another. That is, the transition from one phase to another is not sharp. Figure 5 represents two phases $\mathrm{A}$ and $\mathrm{B}$, in equilibrium, sketched by the colors blue and red, respectively. The concentrations $\varphi$ of $\mathrm{A}$ and $\mathrm{B}$ corresponds to $\varphi_{A}^{e q}$ and $\varphi_{B}^{e q}$, respectively, and there exists a thin region (color gradient) where the concentration $\varphi$ varies gradually between $\varphi_{A}^{e q}$ and $\varphi_{B}^{e q}$. This region is the interface between the phases A and B. In our formulation of the chemical energy $\hat{\psi}^{\text {ch }}$, we allow for microstructure evolution of a system undergoing phase separation processes. The phase separation describes a spontaneous phenomena that occurs at temperatures below the critical one. As a consequence, the system favours the formation of spatial domains rich in either phase. Nevertheless, the chemical energy may also prevent phase separation processes as a result of interfacial interactions. Spinodal decomposition processes can occur, for example, in plagioclase feldspars and the binary system magnetite-ulvospinel (Carpenter, 1981; Lindsley, 1981; Droubay et al., 2011)

Conventionally, the chemical energy can be written as $\hat{\psi}^{c h}=\hat{\psi}^{\varphi}+\hat{\psi}^{s}$ where $\hat{\psi}^{\varphi}$ represents the chemical free energy of the homogeneous system and $\hat{\psi}^{s}$ stands for an interfacial potential which relates the concentration gradients. Herein, we use the definition of $\hat{\psi}^{\text {ch }}$ outlined in Clavijo et al. (2019). This functional extends to multicomponent processes of the classical free energy potential used in the Cahn-Hilliard model (Cahn \& Hilliard, 1958; Elliott \& Garcke, 1997). This generalized Cahn-Hilliard equation models the kinetics of the solid solution and, tracks its microstructure evolution. Figure 6 (a) depicts the homogeneous free energy $\hat{\psi}^{\varphi}$ as a function of the local concentration $\varphi$ for different values of absolute temperature $T$. This potential corresponds to the case where two phases compose the solid, for instance, A and B as depicted by Figure 5 . When the absolute temperature is greater than the critical one (i.e., $T>T_{c}$ ), the potential $\hat{\psi}^{\varphi}$ becomes a convex-downward function of $\varphi$ which renders a homogeneous mix, as there only exists a single stable state located at the minimum value of $\hat{\psi}^{\varphi}$. Therefore, for all possible values of concentration, the homogeneous free energy is stable with respect to phase separation. Alternatively, when the absolute temperatures is below the critical temperature $T<T_{c}$, the homogeneous free energy functional becomes a double-well convex upward function from which two stable coexistent phases emerge where the local minimum value of each well represents the concentration at equilibrium of each phase. Thus, the system favours phase separation. Figure 6 (b) showcases the behaviour of the chemical potential calculated as the derivative of the homogeneous free energy functional $\hat{\psi}^{\varphi}$ with respect to the local concentration $\varphi$. 


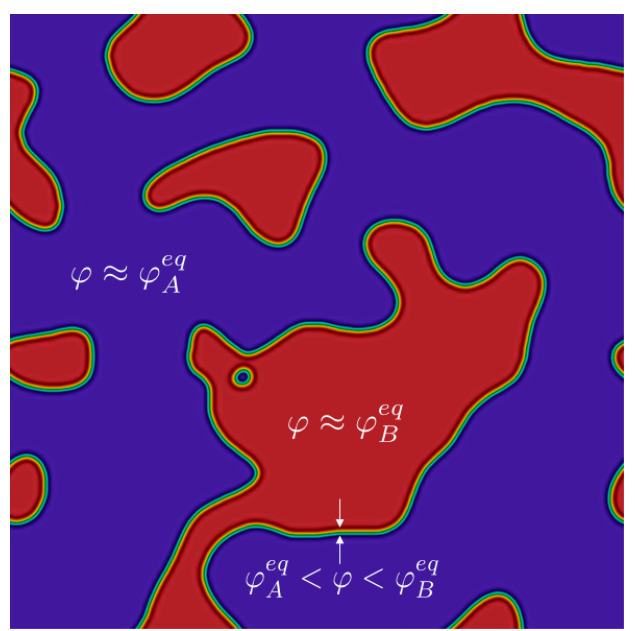

Figure 5 shows the microstructure of a solid solution composed of two phases. The concentration of the phases $\mathrm{A}$ and $\mathrm{B}$ correspond to $\varphi_{A}^{e q}$ and $\varphi_{B}^{e q}$, respectively. The interface, where the concentration varies between $\varphi_{A}^{e q}$ and $\varphi_{B}^{e q}$, embraces the chemical properties of both the phase $\mathrm{A}$ and $\mathrm{B}$.

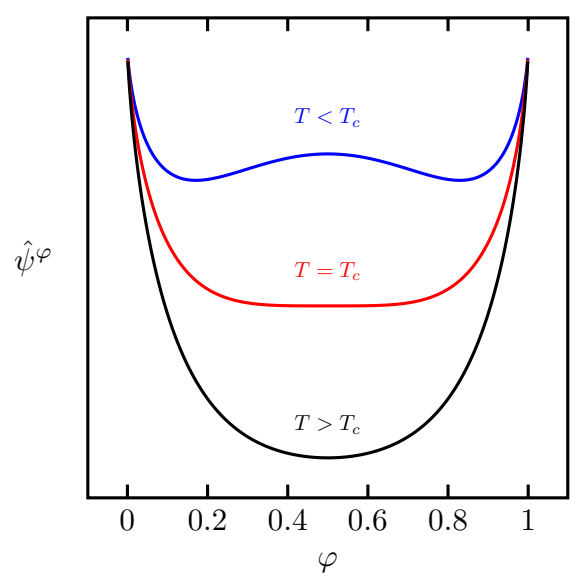

(a)

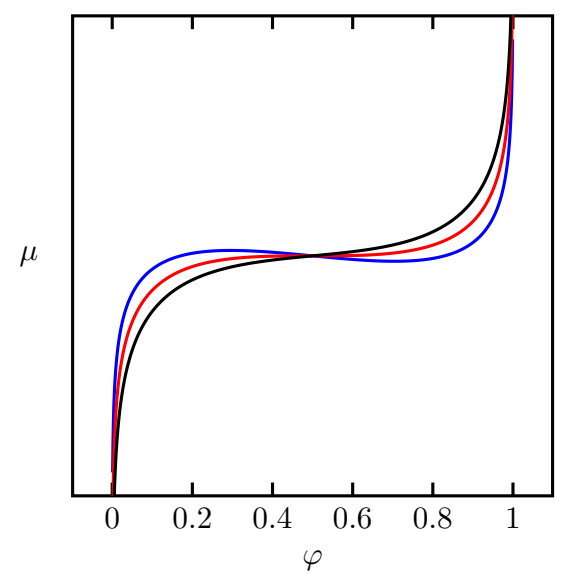

(b)

Figure 6 (a) represents the free energy potential of the homogeneous system. The double well potential function allows for phase separation where the local minimum value of each well accounts for the concentration at equilibrium of each phase, and (b) sketches the chemical potential as a function of the concentration. By definition, the chemical potential is calculated as the partial derivative of the free energy potential with respect to the local concentration.

\subsection{Helmholtz free energy, fundamental balance equations and the im- plied thermodynamic pressure}

The evolution of an elastic solid undergoing chemical processes can be characterized by the total free energy of the system. This energy potential additively accounts for the elastic and chemical energy contributions outlined in Sections 2.3 and 2.4. Hence, 
the total free energy of the system reads

$$
\hat{\Psi}=\hat{\psi}^{c h}+\hat{\psi}^{e l} .
$$

The Helmholtz free energy results from applying the Legendre transform to the internal energy while replacing the entropy of the system by the temperate as an independent variable. To describe the evolution of the solid, we use a set of partial differential equations. These equations describe the balances of mass, and of linear and angular momenta, for the solid-species solution. Moreover, we describe the dynamics of a non-linear elastic solid undergoing phase separation and chemical reaction by subordinating the constitutive relationships to the Helmholtz free energy following the arguments of Coleman \& Noll (1963). We use the aforementioned thermodynamical framework to calculate the chemo-mechanical effects acting on the solid solution and especially to define the thermodynamic pressure that charactirizes the thermodynamic equilibrium.

As mentioned above, the thermodynamic pressure is defined as the partial derivative of the Helmholtz free energy with respect to the specific volume while keeping the local concentration and deformation constant. This physical quantity defines the chemical equilibrium when all dissipative processes, which produce entropy and therefore variations in local composition, cease (Hobbs \& Ord, 2017). Thereby, the thermodynamic pressure defines at the steady state where the free energy potential $\hat{\psi}$ becomes constant (Gibbs, 1878; Hobbs \& Ord, 2017).

\subsection{Thermodynamic pressure in geosystems}

A spike of recent interest in the geosciences literature is the proper definition of the thermodynamic equilibrium in the discussion of the characterization of metamorphic systems. As outlined in Section 1, the thermodynamic pressure can have spatio-temporal inhomogeneities. Recent studies of metamorphic petrology show localized pressure deviations from the lithostatic values that lead to spatially inhomogeneous distributions. Such inhomogeneities arise from the complex chemomechanical interactions between the minerals deep in the Earth. Conventionally, the pressure assumes the Archimedes's value (directly proportional to the depth). Nevertheless, when considering deforming rocks together with mineral reactions, stresses emerge from both volume changes due to reactions and the overburden which lead to inhomogeneous pressure distributions. Thus, in general, the Archimedes's formula is inaccurate for these systems. For instance, the formation of ultrahigh-pressure rocks suggests that pressure does not always translate into depth (Moulas et al., 2013). The understanding of the nature of such deviations is crucial since pressure provides, for instance, a constraint for the description of the dynamics of orogens as well as an indirect measurement of the depth history of the sample. We believe that the roots of such discrepancies are the complex chemo-mechanical interactions as the metamorphic rock complexes evolve towards equilibrium, and that both processes are strongly interdependent. Moulas et al. (2013) provide a comprehensive review of the evidence that metamorphic rocks maintain and record significant pressure deviations from the lithostatic values. During prograde metamorphism, high pressure and temperature conditions induce the formation of garnet porphyroblasts that harbour quartz and coesite inclusions. As the inclusions grow, the metamorphic system endures large volumetric stresses associated with the expansion of the inclusions in the relaxed host matrix. Eventually, the metamorphic system exhibits chemical zonation where each aggregate has different chemical and mechanical properties. Such heterogeneity generates spatial variations in pressure. The effect of the inhomogeneous pressure seems to be critical and will allow the community to better calibrate geodynamics models as well as to describe the evolution of fabrics and microstructures. Previous studies of metamorphic rocks separated the chemical and the mechanical actions on the mineral assemblages. Given the previous discussion, this simplifying splitting is inappropriate as the volume changes, induced by chemical interactions between minerals, strongly influence the inhomogeneous 
pressure distribution. Thus, an appropriate description of the deformation process requires a comprehensive treatment of the coupled chemo-mechanical interactions.

\section{Modeling the effects of inhomogeneous pressure distribution in a ternary solid solution}

To date, a few modeling attempts of the physical and chemical interactions in metamorphic rocks, were made. For instance, Tajčmanová et al. (2015) study the effect of inhomogeneous pressure distributions considering the impact of mass fluxes and external loading to achieve equilibrium while taking into account both pressure gradients and constant pressure. As a consequence, their results suggest that a rock composed of two minerals with different mechanical properties will evolve in such a way that favours mechanically maintained inhomogeneous pressure distributions. Powell et al. (2018) describe nonhydrostatically-stressed metamorphic systems as well as equilibrium conditions at grainboundaries. In their setting, a non-hydrostatically-stressed solid accounts for a solid under inhomogeneous stress distribution. Essentially, their formulation follows the LarchéCahn's framework for multicomponent solids where due to the lattice constrain imposed by the crystalline structure the chemical potentials of the multicomponent system are calculated through the Larché-Cahn derivative (Larché \& Cahn, 1973; Gurtin, 1982; Larché \& Cahn, 1978a). Thus, their proposal leads to a thermodynamic equilibrium for metamorphic systems that accommodates spatial variations in the pressure.

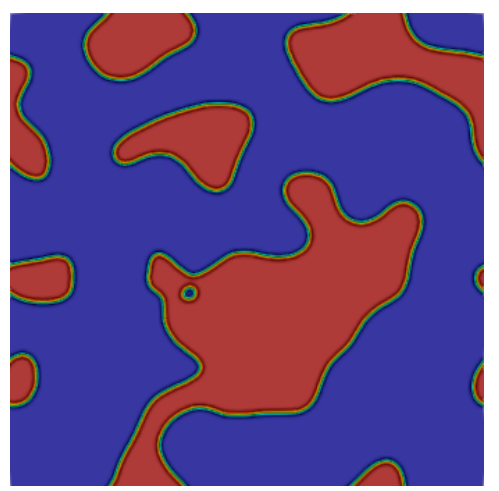

Figure 7 represents the spatial distribution of the initial concentrations for both A and B. Regions colored by red and blue represent the species A and B, respectively. As the chemical and mechanical processes evolve, the system favours generating a new species $\mathrm{C}$ which contributes to the volumetric stress formation in the solid as it nucleates and grows.

In the sequel, we model the dynamics of a solid composed of three phases. We treat the system as a general multicomponent solid whose crystalline structure imposes a mass constraint such that the relation (2.2) holds. One of the phases emerges from a forward chemical reaction. The chemical reaction occurs in solid state, and as it proceeds, the new phase grows and nucleates. As mentioned above, we do not take into account neither diffusion by vacancies nor grain boundaries between the phases. In other words, the crystalline structure of each phase, described by a lattice such as Figure 3, is coherent. We portrait the scenario where local volume changes caused by chemical interactions trigger the stress generation in the solid which in turn reflects spatial variations in pressure. This physical quantity corresponds to the thermodynamic pressure described in Section 2.5. We use the diffusion coefficients, the reaction rates, and the thermodynamic properties of commonly found in solid solutions. Table 1 condenses such quantities. The over- 
all reaction is given by

$$
A+B \stackrel{k}{\rightarrow} C .
$$

Moreover, $\varphi_{1}=[\mathrm{A}], \varphi_{2}=[\mathrm{B}]$ and, $\varphi_{3}=[\mathrm{C}]$ define the concentration of the phases A, $\mathrm{B}$ and $\mathrm{C}$, respectively. Figure 7 depicts the spatial distribution of the initial concentration of each phase. We assume the initial concentration of $\mathrm{C}$ is zero. We also assume a solid without distortions at $t=0.0$. This implies zero relative displacements in the sample and thus, zero strains. We choose a spatial distribution of the concentrations, as Figure 7 shows, to account for large concentration gradients to track the effect of the interface evolution (Cahn \& Hilliard, 1958). Namely, the contribution of the curvature in the chemical potential. We are interested in observing the effect of local variations in the concentration upon the generation of inhomogeneous stress and pressure distributions which relate the species concentration to the deformation gradient as captured by a volumetric stress tensor. Hence, the stress variations come from the volumetric deformation since we do not impose external loading nor deformation. Furthermore, there is no species fluxes through the boundaries. Following Gurtin et al. (2010), the deformation itself cannot induce mass transport. Therefore, for mass transport to happen, there must be chemical potential gradients where the chemo-mechanical coupling accounts for the contributions from both the physical and chemical responses of the solid-species system which in the material sciences literature is known as the absence of a piezo-diffusive effect. We set the chemical energy parameter as well as the number of molecules per volume so that we guarantee a non-convex triple-well energy functional. For instance, Figure 6 models a two-phase case. If required, one can set the chemical energy such that no phase separation emerging from interfacial interactions occur.

Figures 8-11 show the temporal evolution of the concentration of the phases A, B and $\mathrm{C}$ together with the spatial distribution of the thermodynamic pressure, $p^{\text {th }}$, as the solid-three species system evolves to equilibrium. Unlike the thermodynamic pressure, the concentrations and time evolution are presented as dimensionless quantities. As discussed above, we calculate the concentration of the species $C, \varphi_{3}$, by applying at each time-step the constraint defined by 2.2 , which guarantees the consistency of the process. At early stages $t<5.6 \times 10^{-4}$, Figure 8 , the non-Fickian diffusion essentially controls the temporal evolution of both the physical and mechanical processes as the forward chemical reaction plays no substantial role. One can verify such assertion by checking the species mass evolution in Figure 12, where during $t<2.63 \times 10^{-3}$ the species masses remain approximately constant. Moreover, from Figure 8, one can also infer that there is no formation of the species $\mathrm{C}$ until $t>5.6 \times 10^{-4}$. Therefore, the initial condition, spatially distributed as Figure 7, goes through phase separation during the early stages and is followed by coarsening (Clavijo et al., 2019). These interactions lead to a merging process which eventually forms large and rounded structures as suggested by Figures 8 and 9 . Hence, we conclude that at the early stages the source of stress generation is entirely characterised by variations in local composition caused by diffusion and therefore, the dynamics of the spatially inhomogeneous pressure distribution results solely from both the phase separation and coarsening mechanisms while the system seeks to minimise its free energy by reducing the interface between the species A, B and C. Figure 12 also depicts the temporal evolution of the interfacial energy which verifies that during $t<2.63 \times 10^{-3}$, in particular for the species A and B, the interfacial energy decreases. Furthermore, the stress-assisted volume changes mechanism primally occurs along the boundary between $\mathrm{A}$ and $\mathrm{B}$ as the system forms the rounded structures. Namely, large stresses arise along the interface between A and B. Figure 14 reports the dynamics of the neo-Hookean energy functional which captures the shrinkage and swelling process as the phases diffuse through the solid structure. From the free energy evolution, Figure 13, shows that the system is minimising its free energy as the diffusion dissipative process occurs. The minimization is a direct consequence of the principle of minimum energy which states the internal energy is minimised as the system reaches constant entropy. The free energy functional describes the contribution from both the physical and chemical responses of the 
solid. As discussed in Section 2.4, the model captures the dynamics of the Ostwald ripening (Miyazaki, 1991; Eberl et al., 1990; Nemchin et al., 2001). In the range between 1.73x10 ${ }^{-3}<$ $t<2.63 \times 10^{-3}$, Figure 9 illustrates that the smaller aggregates of phase A tend to dissolve into the solid solution and precipitate along the surface of the larger aggregates. Such mechanism leads to a large rounded structure of the species A which is entirely enclosed by the species B. Figure 9 also shows the heterogeneous distribution of the thermodynamic pressure. The thickness of the reaction layer between the phases A and B is irregular, see Figure 9 (c)-(d). Conventionally, during reaction-diffusion processes, one can expect a planar growth of the reaction layer. Nevertheless, when considering reactioninduced stresses and interfacial contributions, the chemical potential becomes also a function of both the surface curvature and the mechanical pressure which leads to an irregular reaction layer of thickness. As a result, the driving force of the diffusion process changes along the reaction layer which induces different diffusion rates at the reaction boundary. The forward chemical reaction occurs mainly during the time interval between $8.02 \times 10^{-3}<$ $t<3.91 \times 10^{-2}$, see Figure 10. Milke et al. (2009) define that positive volume changes involve the creation of space by moving out mass from the reaction site, and thus, the reaction products can grow and accommodate. On the contrary, negative volume changes induce mass transport into the reaction site by consuming the reactant phases. During this stage, the system forms the species $\mathrm{C}$ along the boundary between the species $\mathrm{A}$ and B. Experimental evidence of such behaviour is reported by Milke et al. (2007) whose experiments on the system forstatite (fo) - quartz (qtz) - enstatite (en) produces a reaction rim, mainly composed of enstatite (en), of irregular thickness. They suggest that the nature of this behaviour is due to the mechanical contributions to the chemical potentials resulting from the local volume changes caused by the reaction. Although the mechanical contributions influence the chemical potentials, one must also consider the contributions of surface curvature between the phases as they also alter to a large degree the chemical potentials. This curvature effect is called the Gibbs-Thomson effect (Perez, 2005; Johnson, 1965; Almgren \& Wang, 2000). The mechanism of rim growth as defined by metamorphic petrologists results from the chemical reaction between neighboring minerals (Milke et al., 2009; Keller, 2008). In particular, this process is strongly affected by the mechanical properties of the solid and involves large volume changes that lead to large isotropic stresses.

Eventually, the volumetric stress drives the spatial variations in pressure. Moreover, one can verify from Figure 12 that in the range between $8.02 \times 10^{-3}<t<3.91 \times 10^{-2}$ the species masses change as well as the interfacial energy. Consequently, the masses of the reactant species $\mathrm{A}$ and $\mathrm{B}$ tend to decrease while the reaction product, in this case the species $\mathrm{C}$, increases. At the end of such stage, the system completely consumes the mass of the species $\mathrm{A}$ and as expected the interfacial energy of the species $\mathrm{C}$ increases as the forward reaction generates more species $\mathrm{C}$ and therefore grows the interface. Following Clavijo et al. (2019), the reaction term in the chemical process increases the free energy of the system resulting in the growth trend depicted by Figure 13 in the interval $8.02 \times 10^{-3}<t<3.91 \times 10^{-2}$. Finally, the interleaving between the chemical and mechanical responses of the solid form an elongated structure along the solid primally composed of the species B and surrounded by the species C. Due the large volume changes associated with the chemical process, we can see the stresses all along the solid and thereby the notorious inhomogeneous pressure distribution at the steady state. Figure 13 shows that from $t>7.99 \times 10^{-2}$ all dissipative processes ceased as the free energy remains constant. This final pressure defines which strongly depends on the interactions between the physical and chemical responses of the solid.

The red dots shows in Figure 13 represent the beginning of the processes mentioned above. In particular, one and two account for the beginning of the phase separation and coarsening processes, respectively. Analogously, between three and four the system undergoes the Ostwald ripening effect. Finally, five and six define the action of the forward chemical reaction and the steady state of the solid-species system, respectively. 
Table 1: Physical and Chemical parameters

\begin{tabular}{ccc}
\hline Physical parameter & Value & Name \\
\hline$\vartheta[\mathrm{K}]$ & 1373.15 & Temperature \\
$\vartheta^{c}[\mathrm{~K}]$ & 1500.0 & Critical temperature \\
$D\left[\mathrm{~m}^{2} \mathrm{~s}^{-1}\right]$ & $10^{-20}$ & Diffusion coefficient \\
$K^{+}\left[\mathrm{m}^{2} \mathrm{~s}^{-1}\right]$ & $10^{-14}$ & Reaction rate \\
$\sigma\left[\mathrm{J} \mathrm{m}^{-2}\right]$ & 0.817 & Interfacial energy \\
$\ell[\mathrm{m}]$ & $10^{-8}$ & Interface thickness \\
$\mu[\mathrm{GPa}]$ & 44 & Shear modulus \\
$\nu[-]$ & 0.17 & Poisson's ratio \\
$\omega[-]$ & $10^{-2}$ & Swelling parameter \\
\hline
\end{tabular}
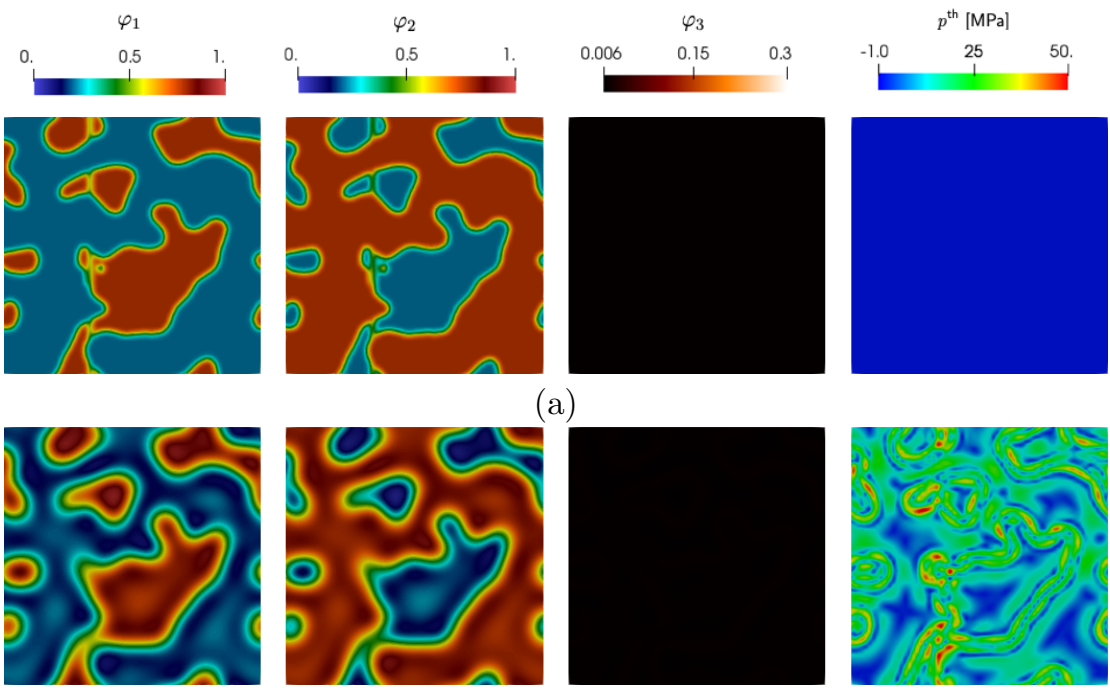

(a)
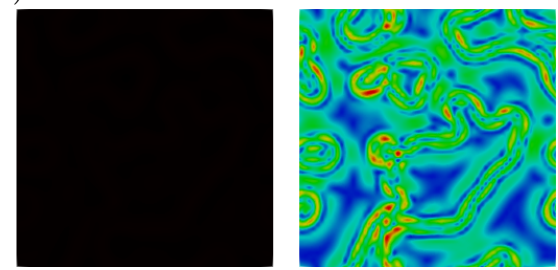

(b)
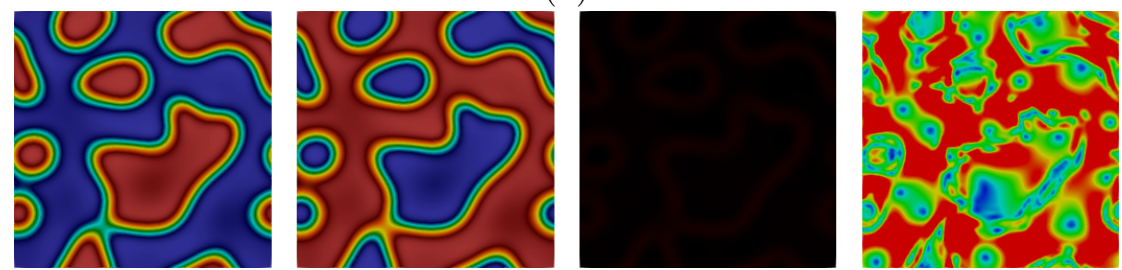

(c)
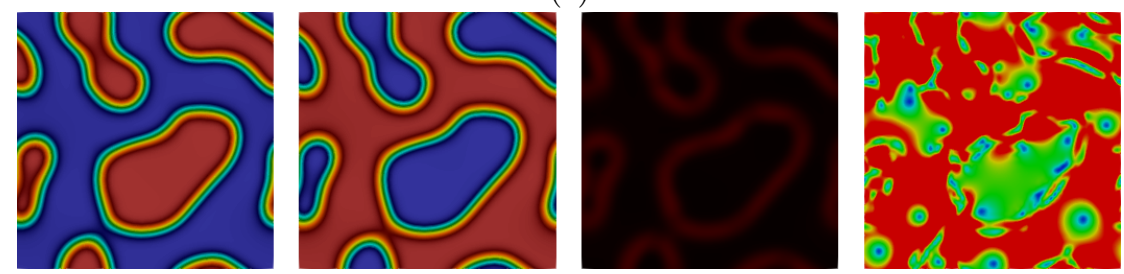

(d)

Figure 8 depicts the behaviour of the three phases system at the early stages. According to the evolution, the system is mostly controlled by the interleaving of phase separation and coarsening. Therefore, the volume changes and subsequent stress generation result from the diffusion process itself leading to the inhomogeneous pressure distribution. 

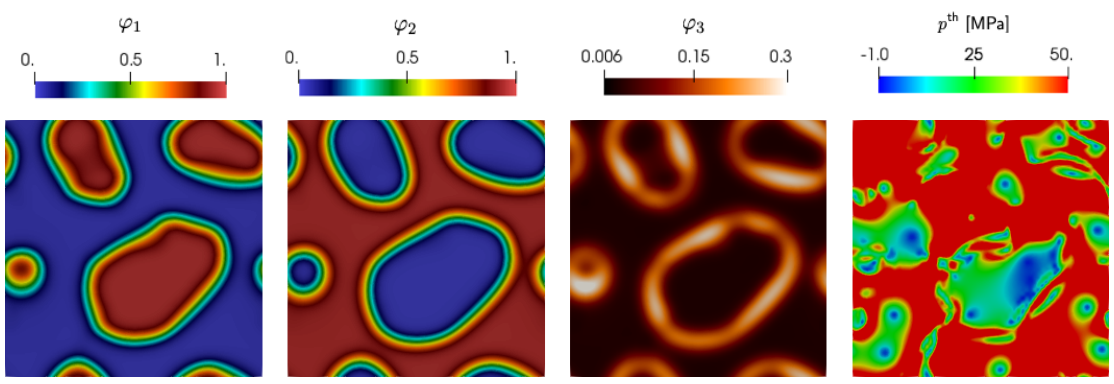

(a)
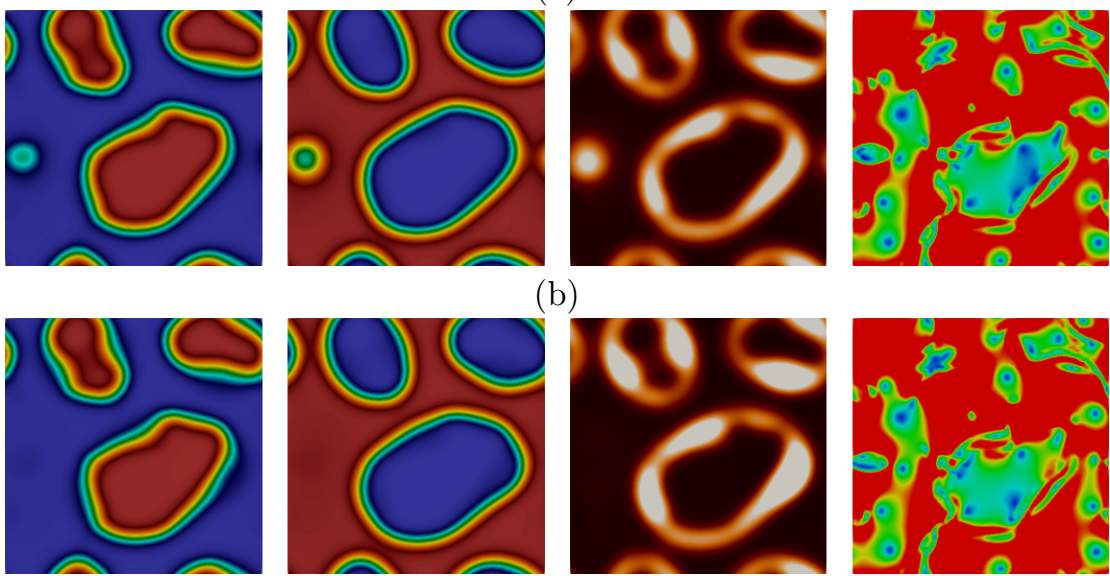

(b)
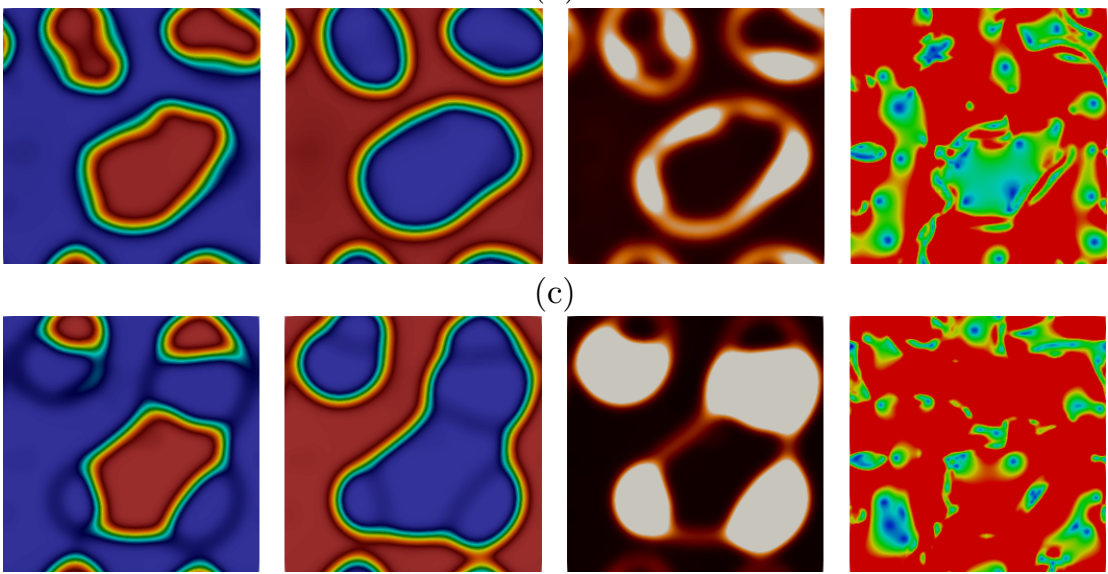

(c)
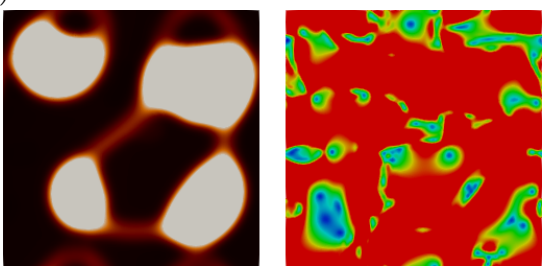

(d)

Figure 9 portraits the dynamics of the system as it follows the Ostwald ripening effect. The unstable particles on the surface dissolve and go into the solution, and once the solution gets supersaturated, these particles tend to precipitate onto the surface of the more stable structures. Consequently, the larger structure in the system grows. 

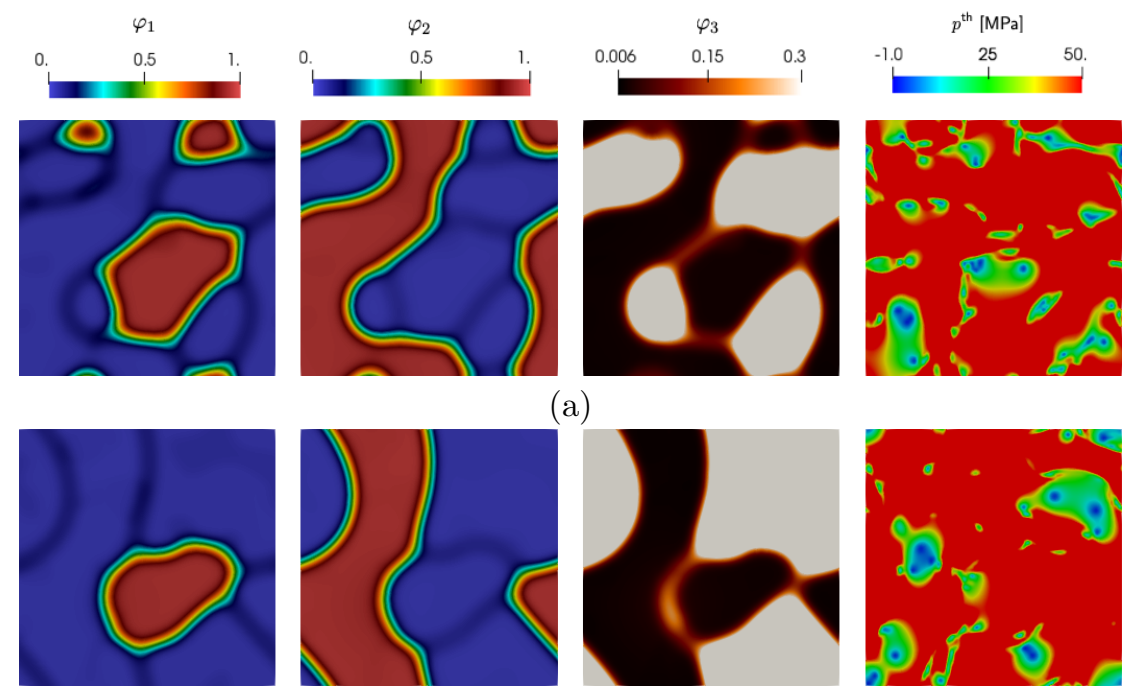

(a)
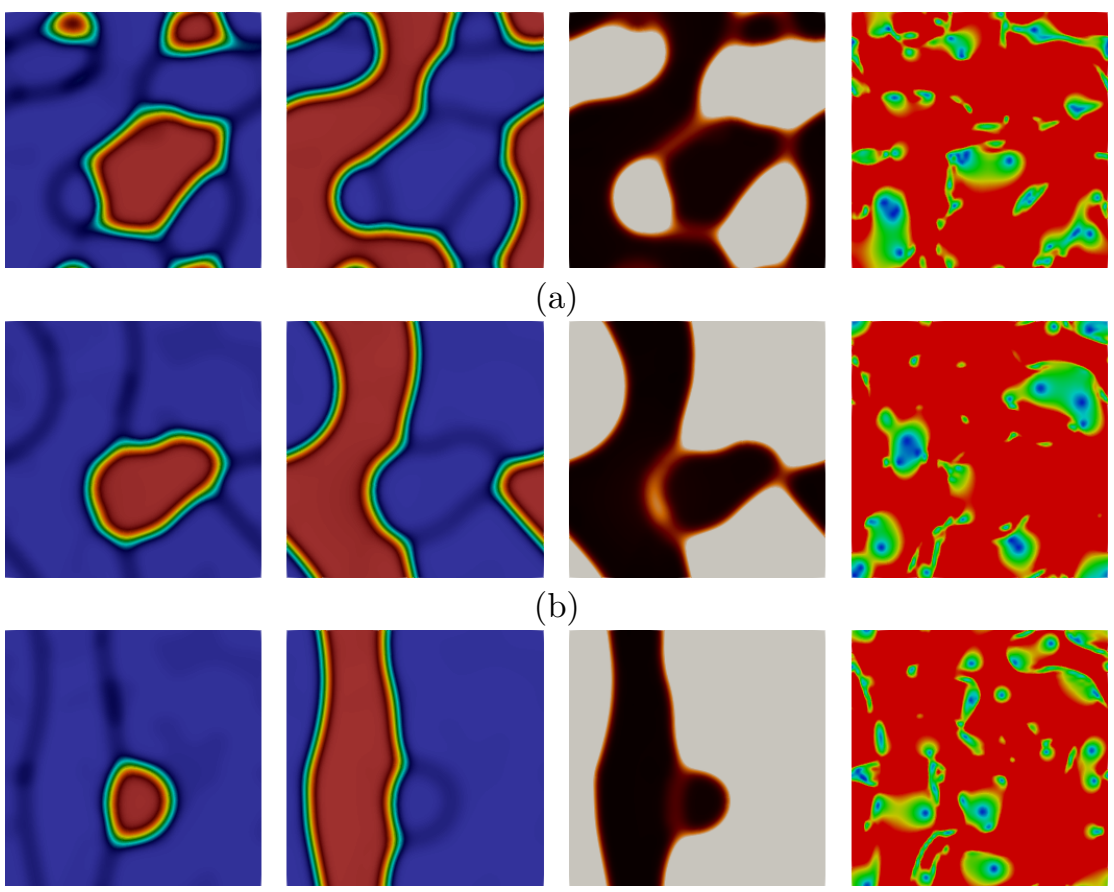

(b)
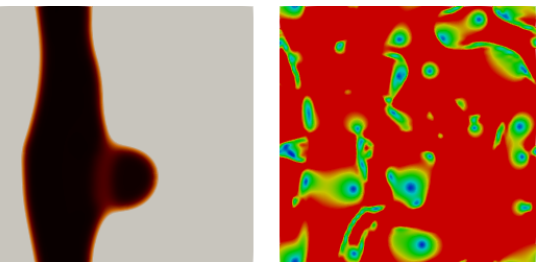

(c)
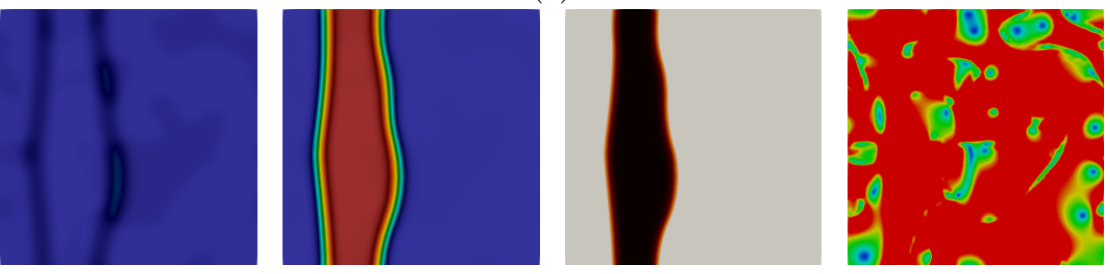

(d)

Figure 10 shows the reaction between the species $\mathrm{A}$ and $\mathrm{B}$ to produce a new phase $\mathrm{C}$ along their boundary. The evolution favours to consume in a greater proportion the phase A than the phase B. The simulation results show how the nucleation and growth of phases induce volumetric stresses which in turn contribute to generate the inhomogeneous pressure distribution. 

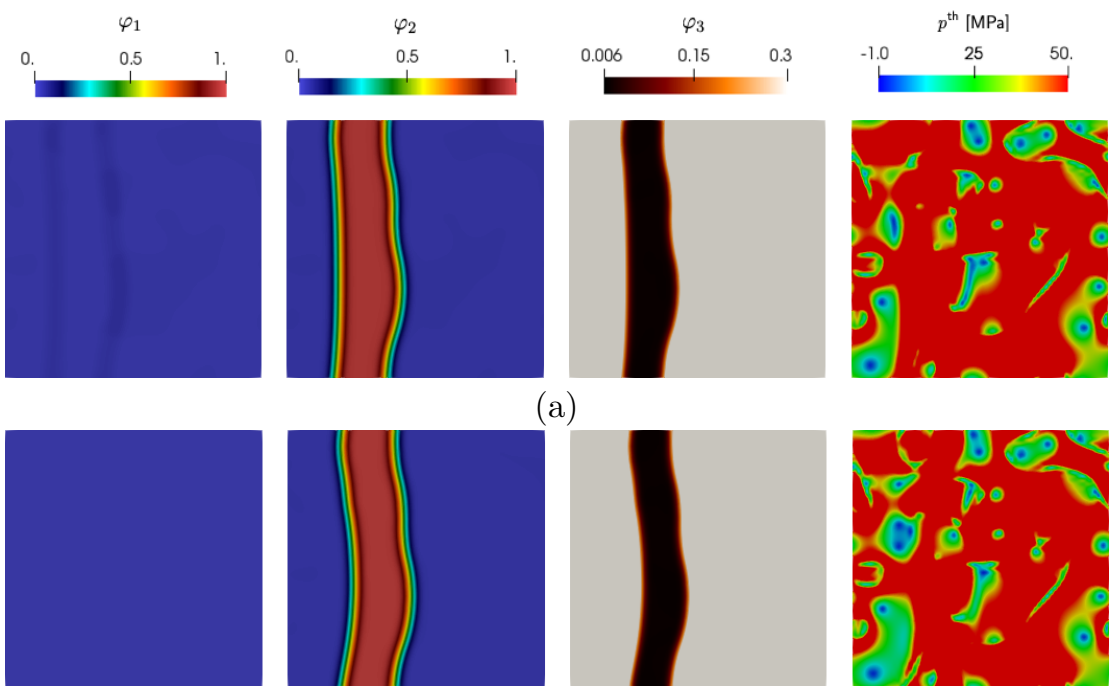

(a)
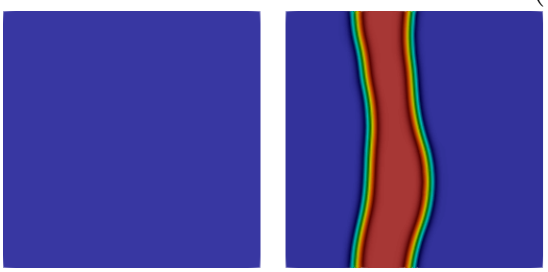

(b)
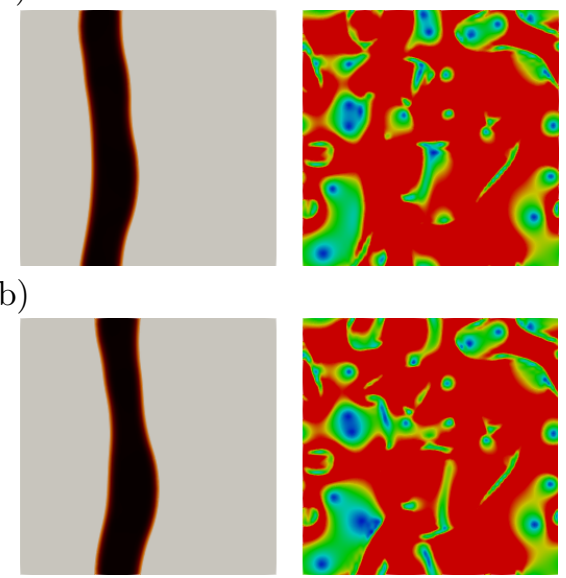

(c)
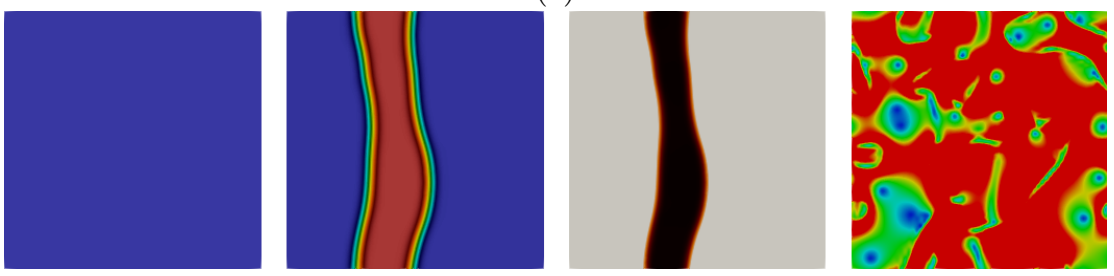

(d)

Figure 11 suggests that the chemical reaction acts as either a source or sink of energy. In this particular case, it contributes to increasing the free energy of the system. Once the chemical process ceases, the system minimizes its free energy solely by mass transport leading to the steady state at $t>9.33 \times 10^{4}$. Finally, the thermodynamic pressure at $t>9.33 \times 10^{4}$, which results from the contribution of both chemical and mechanical responses of the solid, defines the equilibrium of the metamorphic system. 

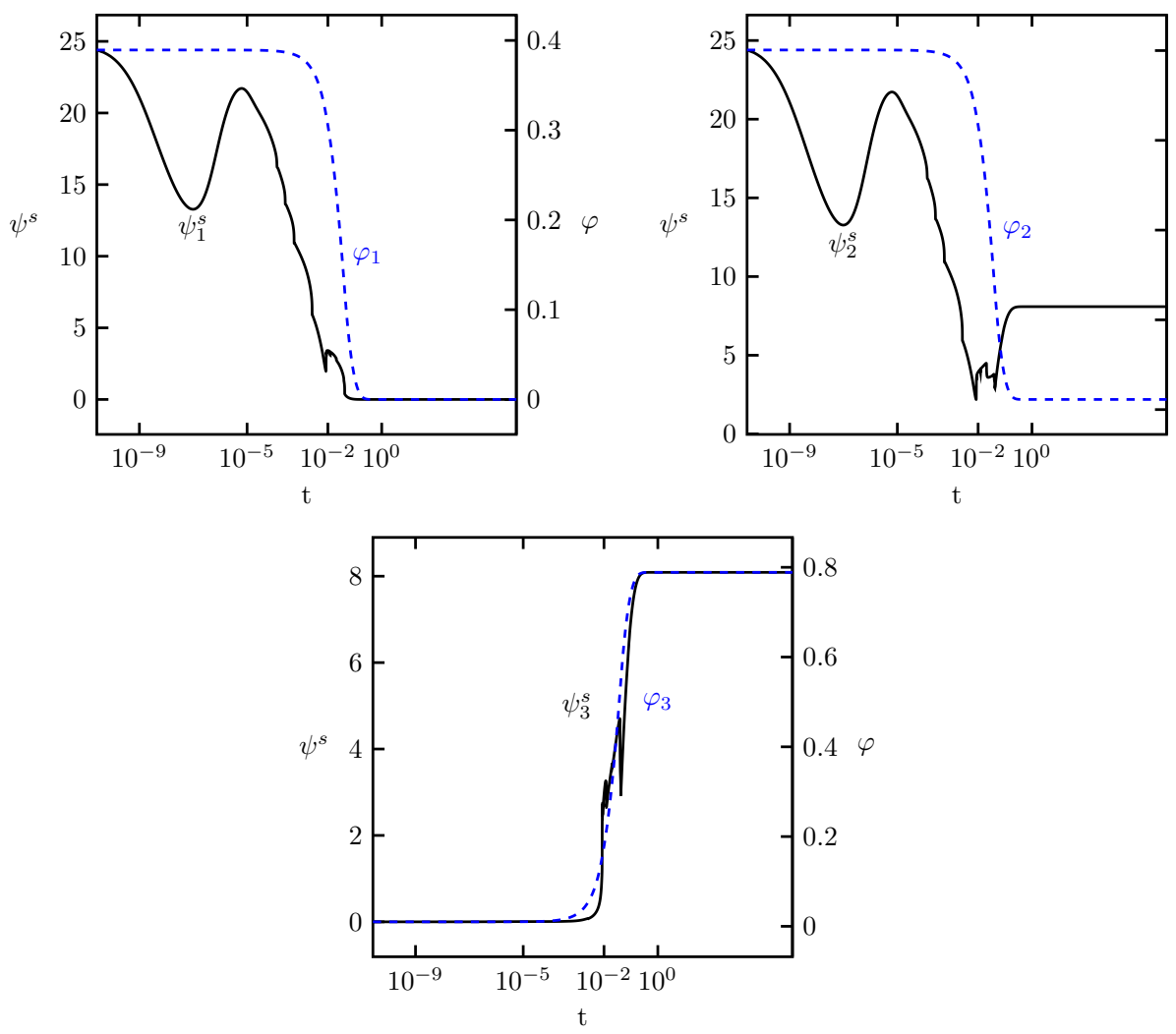

Figure 12 depicts that when a system undergoes a chemical process, either mass transport or chemical reaction, the dynamics favours to either produce or destroy the interface between the species. Thus, the interfacial energy must change according to this evolution process. 


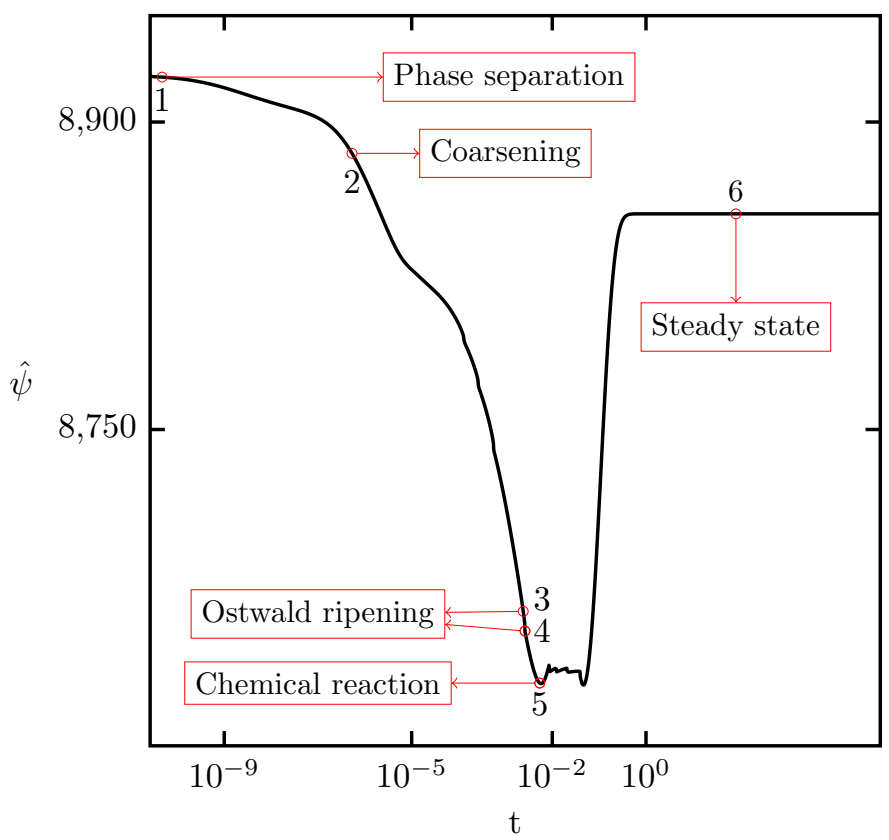

Figure 13 shows the free energy evolution and marks the beginning of processes such as phase separation and coarsening, Ostwald ripening effect, the chemical reaction and steady state.

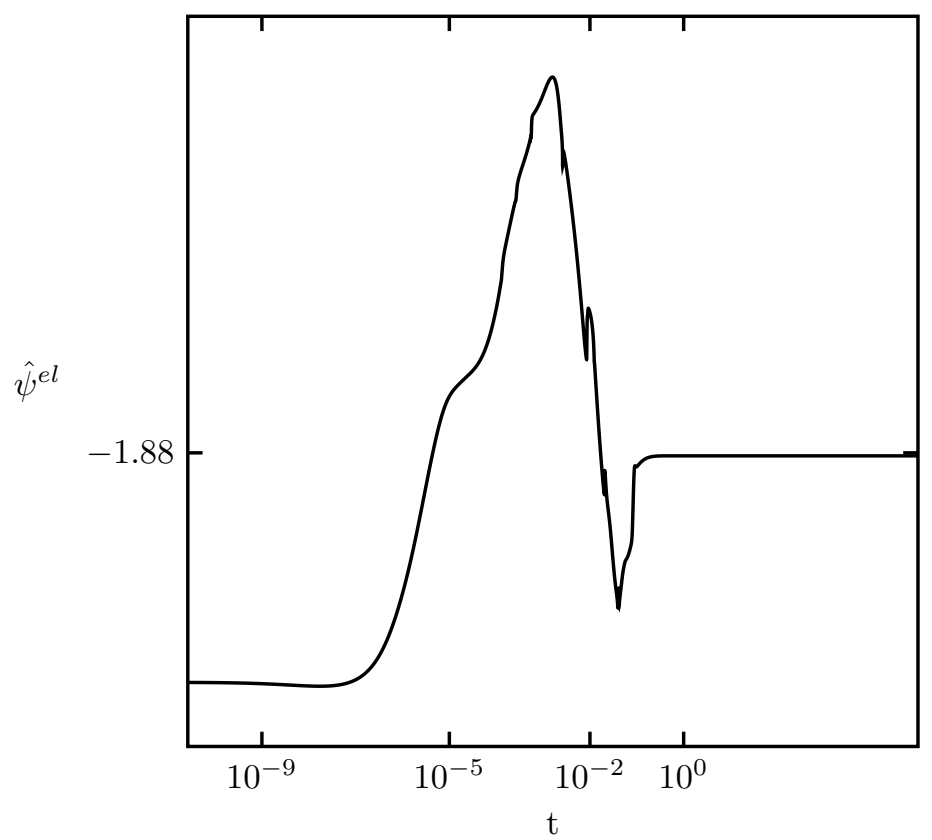

Figure 14 depicts the elastic energy of a neo-Hookean solid model. Since no deformation is induced across the solid boundaries, the variation of the elastic energy entirely results from the volumetric stresses associated with the variations in local composition. 
The possibility of describing the formation of metamorphic mineral aggregates with spatial inhomogeneous pressure distributions collides with the classical description of the formation of metamorphic minerals. The classical interpretations assume an isotropic thermodynamic equilibrium to explain metamorphic mineral assemblages via thermobarometry techniques and phase diagrams. Therefore, the formation processes that induce heterogeneous pressure distributions imply that these techniques may not be robust to characterize metamorphic systems. That is, these inhomogeneous pressure conditions contradict the foundational assumptions of uniform pressure and temperature distributions. Thus, the nature of the pressure distribution that defines the equilibrium of metamorphic rocks and especially how to calculate and define this quantity are still open questions in our opinion. As pointed out by Hobbs \& Ord (2016) (and references therein), the thermodynamic equilibrium is entirely characterized by the thermodynamic pressure given by the partial derivative of the Helmholtz free energy with respect to the specific volume or when considering Gibbs free energy, the partial derivative has to be taken with respect to the volume. We believe that heretofore, in the geosciences literature, the lithostatic pressure has erroneously been used to describe the state of equilibrium of the metamorphic rocks. And recently, works on inhomogeneous pressure distributions use the mean stress to characterize equilibrium conditions (Tajčmanová et al., 2015, 2014). Such pressure definitions only make sense form a thermodynamic point of view when the solid behaves elastically without ongoing chemical processes (Hobbs \& Ord, 2016). Solids under either viscoelastic, diffusional creep, or plastic behavior as well as ongoing chemical reactions between the constituent phases relate dissipative processes which lead to additional contributions to the definition of thermodynamic pressure. Hobbs \& Ord $(2016,2017)$ have carried out an extensive review on the subject (see also e.g Bennethum \& Weinstein (2004) and references therein).

\section{Conclusions}

Using a chemo-mechanical model for solid solutions, we study how the mechanical response affects on the evolution of a chemically active solid-three species solution. We demonstrate that the interleaving between the chemical and mechanical responses of the multicomponent solid influences the generation of spatial variations in pressure. This pressure corresponds to the thermodynamic pressure and defines the equilibrium conditions of the system. By setting the corresponding physical and mechanical properties, one can model the chemo-mechanical behaviour of a multicomponent solid which follows interfacial effects as well as large stresses. Moreover, this framework can help as a first step to model the behaviour of the stress-generation processes in metamorphic minerals which lead to spatial distribution of the thermodynamic pressure. Nevertheless, one must beard in mind that, phase separation mechanisms are not common processes in metamorphic systems. Therefore, to model these metamorphic systems using the aforementioned framework, the chemical energy must set such that no phase separation takes place along the process. This can be achieved by choosing the initial distribution of the phases concentration at the minimum values of the chemical energy. Thereby, the system does not tend to minimise the energy by separating the phases.

\section{Acknowledgments}

This publication was also made possible in part by the CSIRO Professorial Chair in Computational Geoscience at Curtin University and the Deep Earth Imaging Enterprise Future Science Platforms of the Commonwealth Scientific Industrial Research Organisation, CSIRO, of Australia. This project has received funding from the European Union's Horizon 2020 research and innovation programme under the Marie Sklodowska-Curie grant agreement No 777778 (MATHROCKS). At Curtin University, The Institute for Geoscience Research (TIGeR) and by the Curtin Institute for Computation, kindly provide continuing support. The data can be found in "Clavijo, S.P. (Santiago Pea) (2020) Data un- 
derlying the Numerical simulation of a three-phase system using a coupled reactive chemomechanical model. 4TU.Centre for Research Data. Dataset. https://doi.org/10.4121/uuid:299c1e20a606-4ed6-9fe2-ba0283df39d6".

\section{References}

Almgren, F., \& Wang, L. (2000). Mathematical existence of crystal growth with gibbs-thomson curvature effects. The Journal of Geometric Analysis, 10(1), 1.

Bennethum, L. S., \& Weinstein, T. (2004). Three pressures in porous media. Transport in Porous Media, 54(1), 1-34.

Cahn, J. W., \& Hilliard, J. E. (1958). Free energy of a nonuniform system. i. interfacial free energy. The Journal of chemical physics, 28(2), 258-267.

Carpenter, M. A. (1981). A conditional spinodal within the peristerite miscibility gap of plagioclase feldspars. American Mineralogist, 66 (5-6), 553-560.

Clavijo, S., Sarmiento, A., Espath, L., Dalcin, L., Cortes, A., \& Calo, V. (2019). Reactive n-species cahn-hilliard system: A thermodynamically-consistent model for reversible chemical reactions. Journal of Computational and Applied Mathematics, 350, 143-154.

Coleman, B. D., \& Noll, W. (1963). The thermodynamics of elastic materials with heat conduction and viscosity. Archive for rational mechanics and analysis, 13(1), $167-178$.

Dal, H., \& Miehe, C. (2015). Computational electro-chemo-mechanics of lithium-ion battery electrodes at finite strains. Computational Mechanics, 55(2), 303-325.

Droubay, T. C., Pearce, C. I., Ilton, E. S., Engelhard, M. H., Jiang, W., Heald, S. M., ... Rosso, K. M. (2011). Epitaxial fe 3- x ti x o 4 films from magnetite to ulvöspinel by pulsed laser deposition. Physical Review B, 84 (12), 125443.

Eberl, D. D., Środoń, J., Kralik, M., Taylor, B. E., \& Peterman, Z. E. (1990). Ostwald ripening of clays and metamorphic minerals. Science, 248(4954), 474-477.

Elliott, C. M., \& Garcke, H. (1997). Diffusional phase transitions in multicomponent systems with a concentration dependent mobility matrix. Physica D: Nonlinear Phenomena, $109(3-4), 242-256$.

Gibbs, J. W. (1878). On the equilibrium of heterogeneous substances. American Journal of Science(96), 441-458.

Gurtin, M. E. (1982). An introduction to continuum mechanics (Vol. 158). Academic press.

Gurtin, M. E., Fried, E., \& Anand, L. (2010). The mechanics and thermodynamics of continua. Cambridge University Press.

Hobbs, B. E., \& Ord, A. (2016). Does non-hydrostatic stress influence the equilibrium of metamorphic reactions? Earth-Science Reviews, 163, 190-233.

Hobbs, B. E., \& Ord, A. (2017). Coupling of fluid flow to permeability development in mid-to upper crustal environments: a tale of three pressures. Geological Society, London, Special Publications, 453, SP453-9.

Howell, D., Wood, I., Dobson, D., Jones, A., Nasdala, L., \& Harris, J. (2010). Quantifying strain birefringence halos around inclusions in diamond. Contributions to Mineralogy and Petrology, 160(5), 705-717.

Johnson, C. A. (1965). Generalization of the gibbs-thomson equation. Surface Science, 3(5), 429-444.

Keller, L. (2008). Mineral growth in metamorphic rocks: relationships between chemical patterns, mineral microstructure and reaction kinetics. In Agu fall meeting abstracts.

Larché, F., \& Cahn, J. W. (1973). A linear theory of thermochemical equilibrium of solids under stress. Acta metallurgica, 21 (8), 1051-1063.

Larché, F., \& Cahn, J. W. (1978a). A nonlinear theory of thermochemical equilibrium of solids under stress. Acta Metallurgica, 26(1), 53-60. 
Larché, F., \& Cahn, J. W. (1978b). Thermochemical equilibrium of multiphase solids under stress. Acta Metallurgica, 26(10), 1579-1589.

Larché, F., \& Cahn, J. W. (1984). The interactions of composition and stress in crystalline solids. J. Res. Nat. Bur. Stand., 89(6), 467.

Lindsley, D. H. (1981). Some experiments pertaining to the magnetite-ulvöspinel miscibility gap. American Mineralogist, 66 (7-8), 759-762.

Miehe, C., Dal, H., Schänzel, L.-M., \& Raina, A. (2016). A phase-field model for chemo-mechanical induced fracture in lithium-ion battery electrode particles. International Journal for Numerical Methods in Engineering, 106(9), 683-711.

Milke, R., Abart, R., Kunze, K., KOCH-MÜLLER, M., Schmid, D., \& Ulmer, P. (2009). Matrix rheology effects on reaction rim growth i: evidence from orthopyroxene rim growth experiments. Journal of Metamorphic Geology, 27(1), 71-82.

Milke, R., Dohmen, R., Becker, H.-W., \& Wirth, R. (2007). Growth kinetics of enstatite reaction rims studied on nano-scale, part i: Methodology, microscopic observations and the role of water. Contributions to Mineralogy and Petrology, $154(5), 519-533$.

Miyazaki, K. (1991). Ostwald ripening of garnet in high $\mathrm{p} / \mathrm{t}$ metamorphic rocks. Contributions to Mineralogy and Petrology, 108(1-2), 118-128.

Miyazaki, K. (1996). A numerical simulation of textural evolution due to ostwald ripening in metamorphic rocks: A case for small amount of volume of dispersed crystals. Geochimica et cosmochimica acta, 60(2), 277-290.

Moulas, E., Podladchikov, Y., Aranovich, L. Y., \& Kostopoulos, D. (2013). The problem of depth in geology: When pressure does not translate into depth. Petrology, $21(6), 527-538$.

Nemchin, A., Giannini, L., Bodorkos, S., \& Oliver, N. (2001). Ostwald ripening as a possible mechanism for zircon overgrowth formation during anatexis: theoretical constraints, a numerical model, and its application to pelitic migmatites of the tickalara metamorphics, northwestern australia. Geochimica et Cosmochimica Acta, 65(16), 2771-2788.

Perez, M. (2005). Gibbs-thomson effects in phase transformations. Scripta materialia, 52(8), 709-712.

Powell, R., Evans, K. A., Green, E. C., \& White, R. W. (2018). On equilibrium in non-hydrostatic metamorphic systems. Journal of Metamorphic Geology, 36(4), 419-438.

Sekerka, R. F., \& Cahn, J. W. (2004). Solid-liquid equilibrium for non-hydrostatic stress. Acta materialia, 52(6), 1663-1668.

Tajčmanová, L., Podladchikov, Y., Powell, R., Moulas, E., Vrijmoed, J., \& Connolly, J. (2014). Grain-scale pressure variations and chemical equilibrium in high-grade metamorphic rocks. Journal of Metamorphic Geology, 32(2), 195-207.

Tajčmanová, L., Vrijmoed, J., \& Moulas, E. (2015). Grain-scale pressure variations in metamorphic rocks: implications for the interpretation of petrographic observations. Lithos, 216, 338-351.

Truesdell, C. (1984). Rational thermodynamics. Springer.

Tsagrakis, I., \& Aifantis, E. C. (2017). Thermodynamic coupling between gradient elasticity and a Cahn-Hilliard type of diffusion: size-dependent spinodal gaps. Continuum Mechanics and Thermodynamics, 1-14.

Vrijmoed, J. C., \& Podladchikov, Y. Y. (2015). Thermodynamic equilibrium at heterogeneous pressure. Contributions to Mineralogy and Petrology, 170(1), 10.

Wheeler, J. (2014). Dramatic effects of stress on metamorphic reactions. Geology, 42(8), 647-650.

Zhong, X., Vrijmoed, J., Moulas, E., \& Tajčmanová, L. (2017). A coupled model for intragranular deformation and chemical diffusion. Earth and Planetary Science Letters, 474, 387-396. 
Figure 1.

(n) 
ESSOAr | https://doi.org/10.10
Figure 2. 


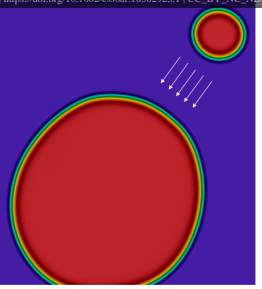

(a)

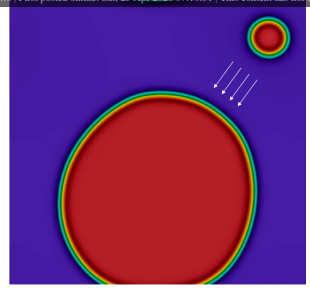

(b)

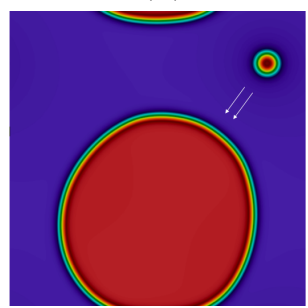

(d)

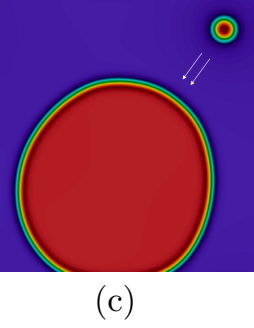


ESSOAr | https://doi.or
Figure 3.

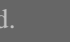




\section{ESSOAr | https://doi.org/10.1002/essoar.10502923.1 | CC_BY_NC_ND_4.0 | First posted online: Sat, 25 Apr 2020 07:10:31 | This content has not been peer reviewed.}

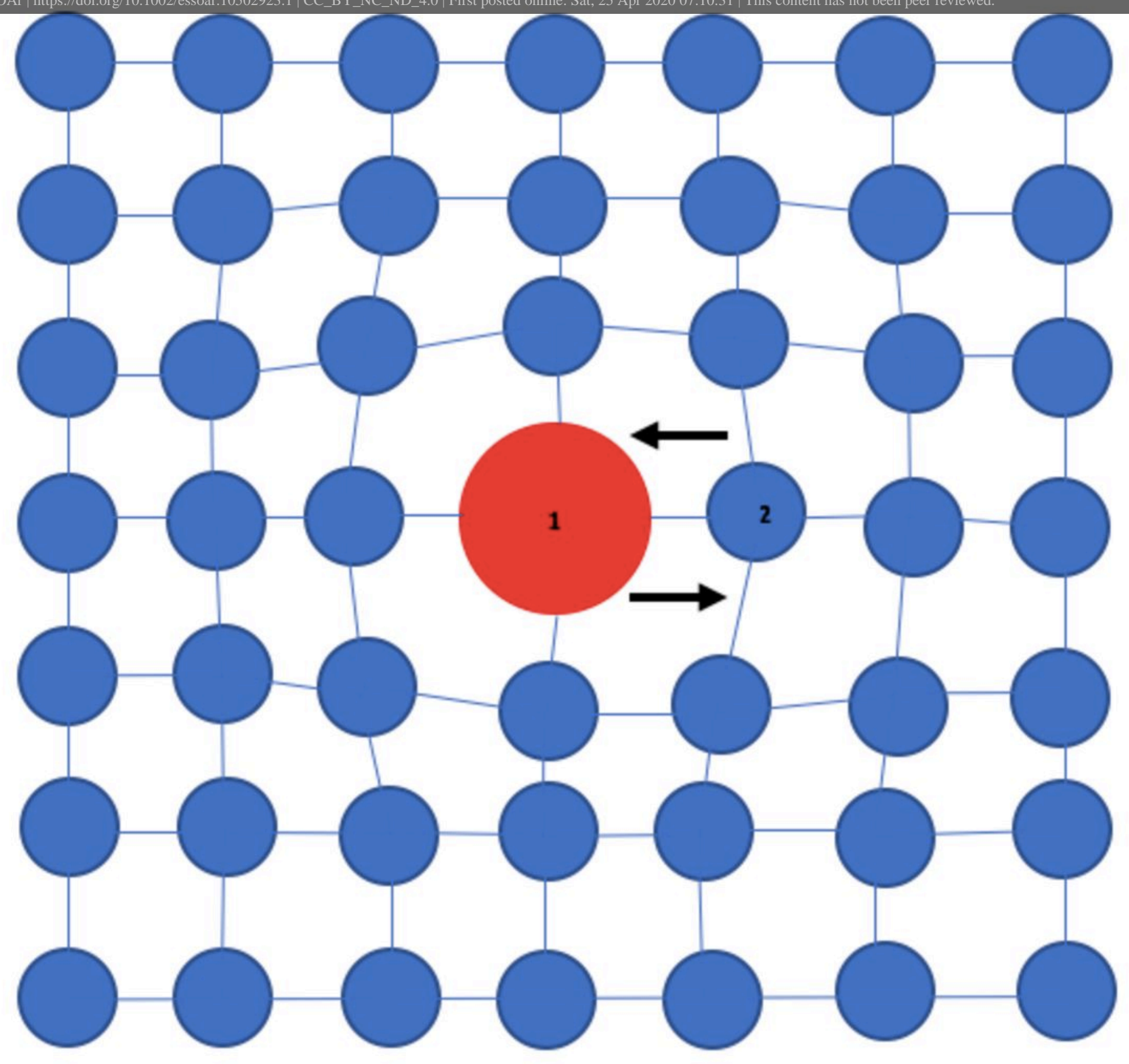


Figure 4. 


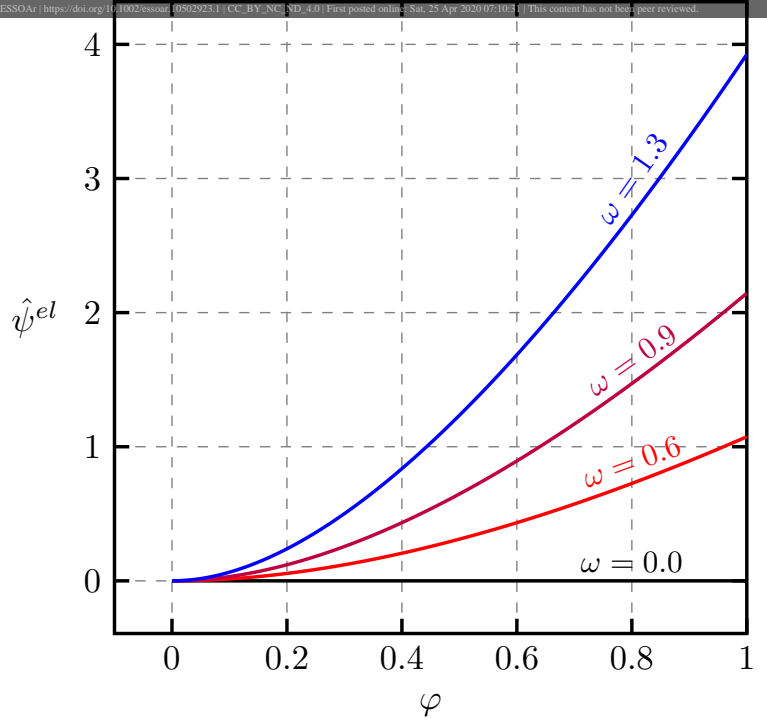


Figure 5. 


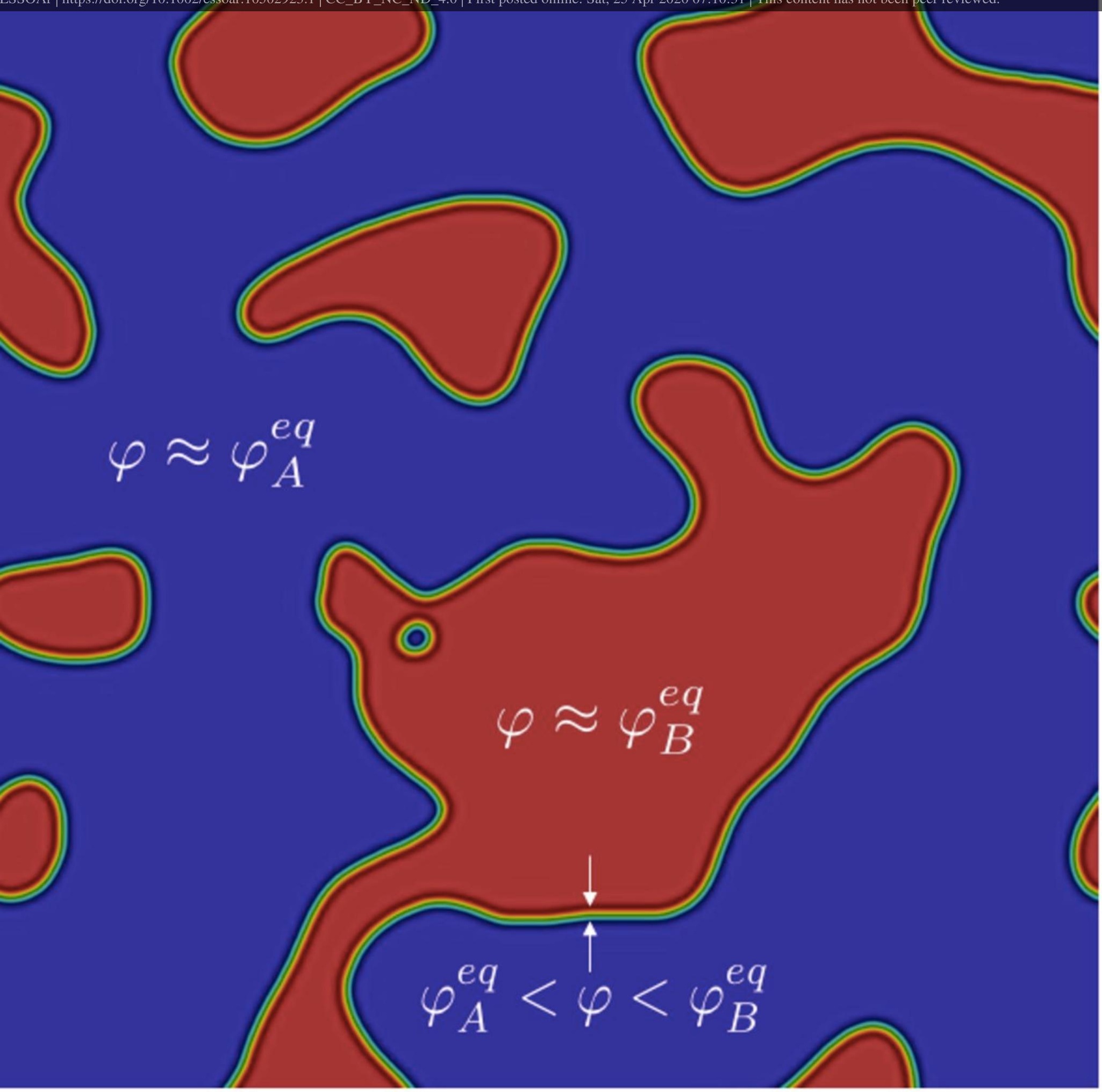


Figure 6.

ESSOAr $\mid$ https://do

.

6. 
Figure 7. 

Figure 8.

Figure 8. 
Figure 9. 
Figure 10.

ESSOAr | https://doi.or

(n)$$
\text { d. }
$$

更 
ESSOAr | hups://doi.org/10.1002/essoar.10502923.1 | CC_BY_NC_ND_4.0 | First posted online: Sat, 25 Apr 2020 07:10:31 | This content has not been peer reviewed.
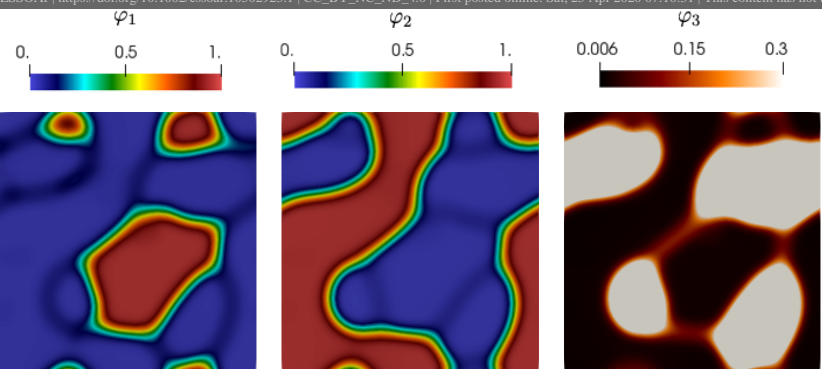

$-1.0$

$\left.p^{\text {th }} \mid \mathrm{MPa}\right]$

25

50.

1

(a)
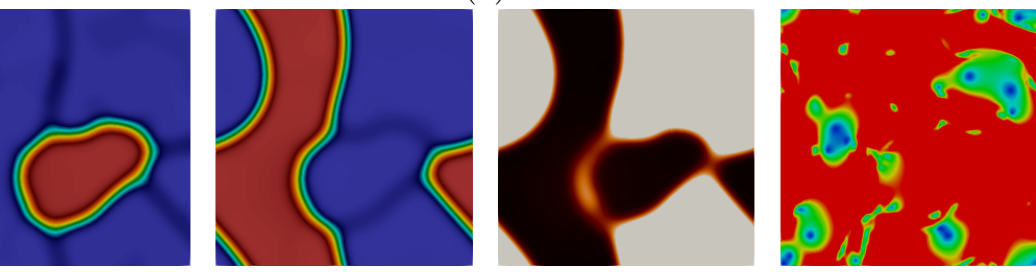

(b)
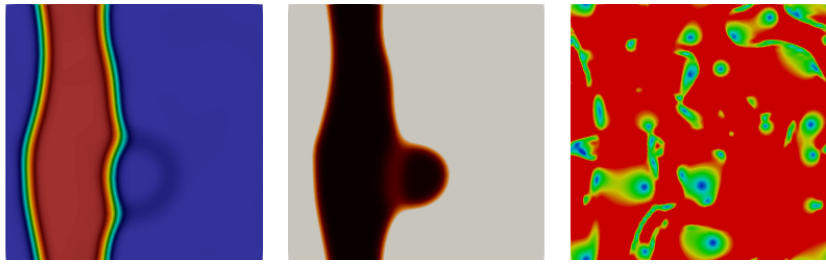

(c)
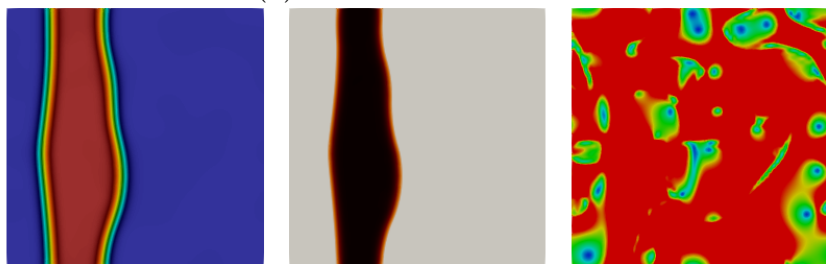

(d) 
ESSOAr | https://doi.c

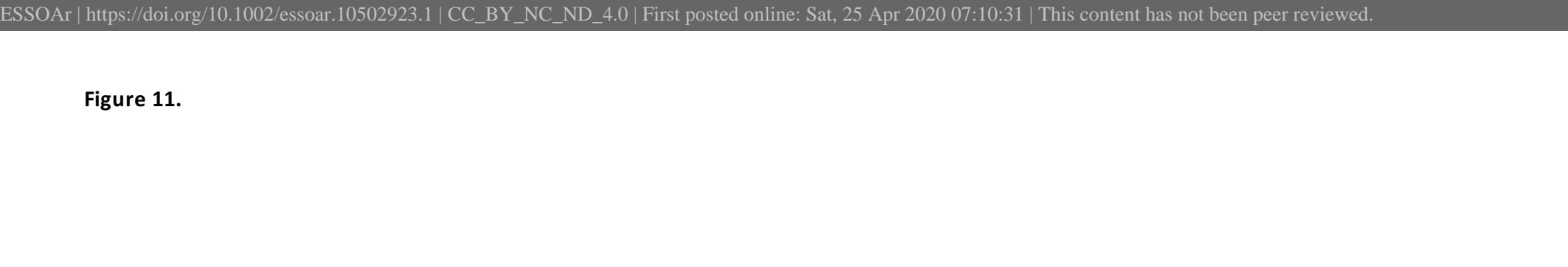

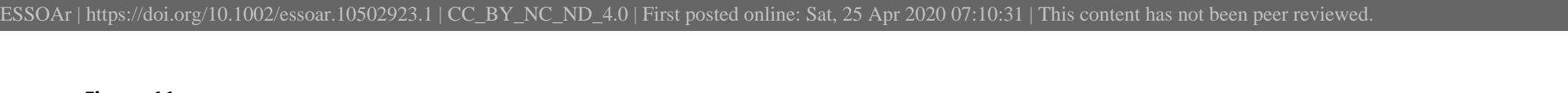
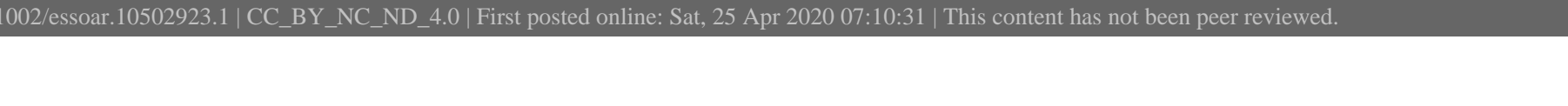

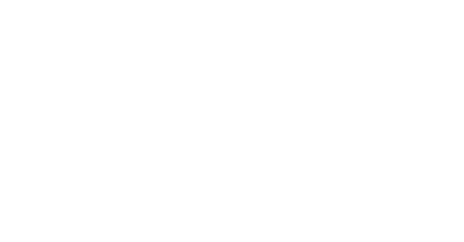

Figure 11.

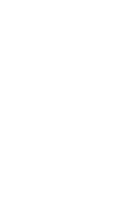

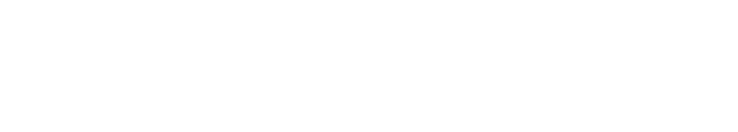

\section{Figure 11. \\ (n)}

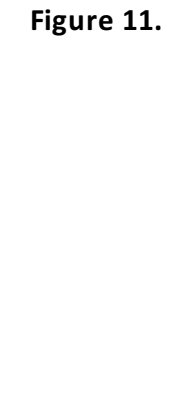

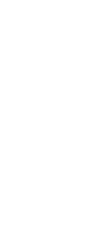

rats 
ESSOAr | hups://doi.org/10.1002/essoar:10502923.1 | CC_BY_NC_ND_4.0 | First posted online: Sat, 25 Apr 2020 07:10:31 | This content has not been peer reviewed.
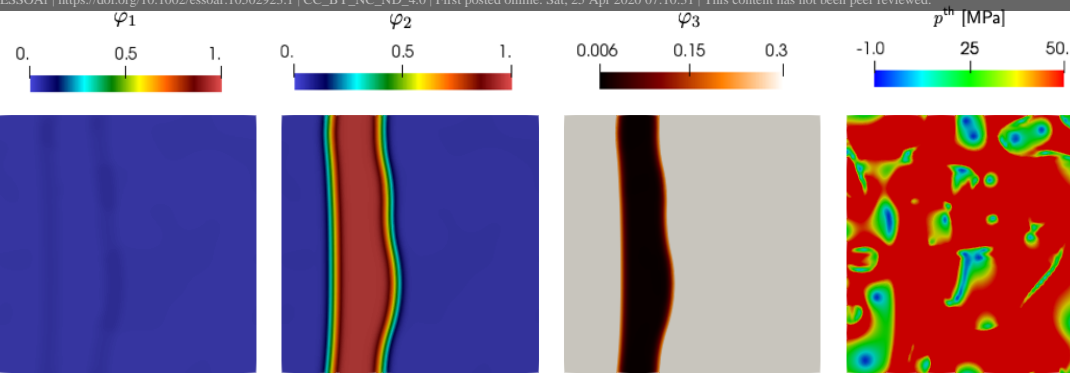

(a)
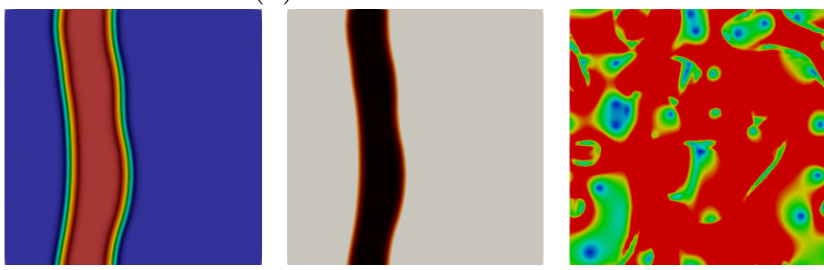

(b)
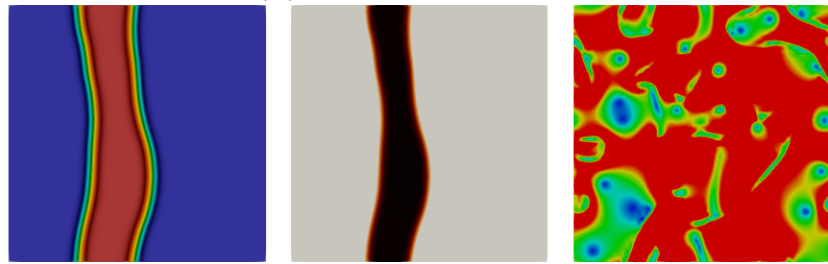

(c)
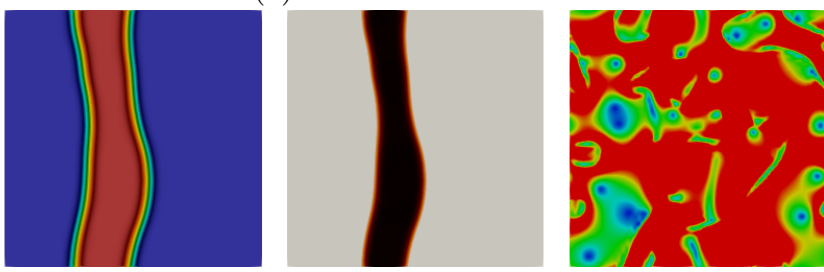

(d) 
ESSOAr | https://doi.or

(n)

Figure 12. 10.1002/essoar.

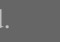



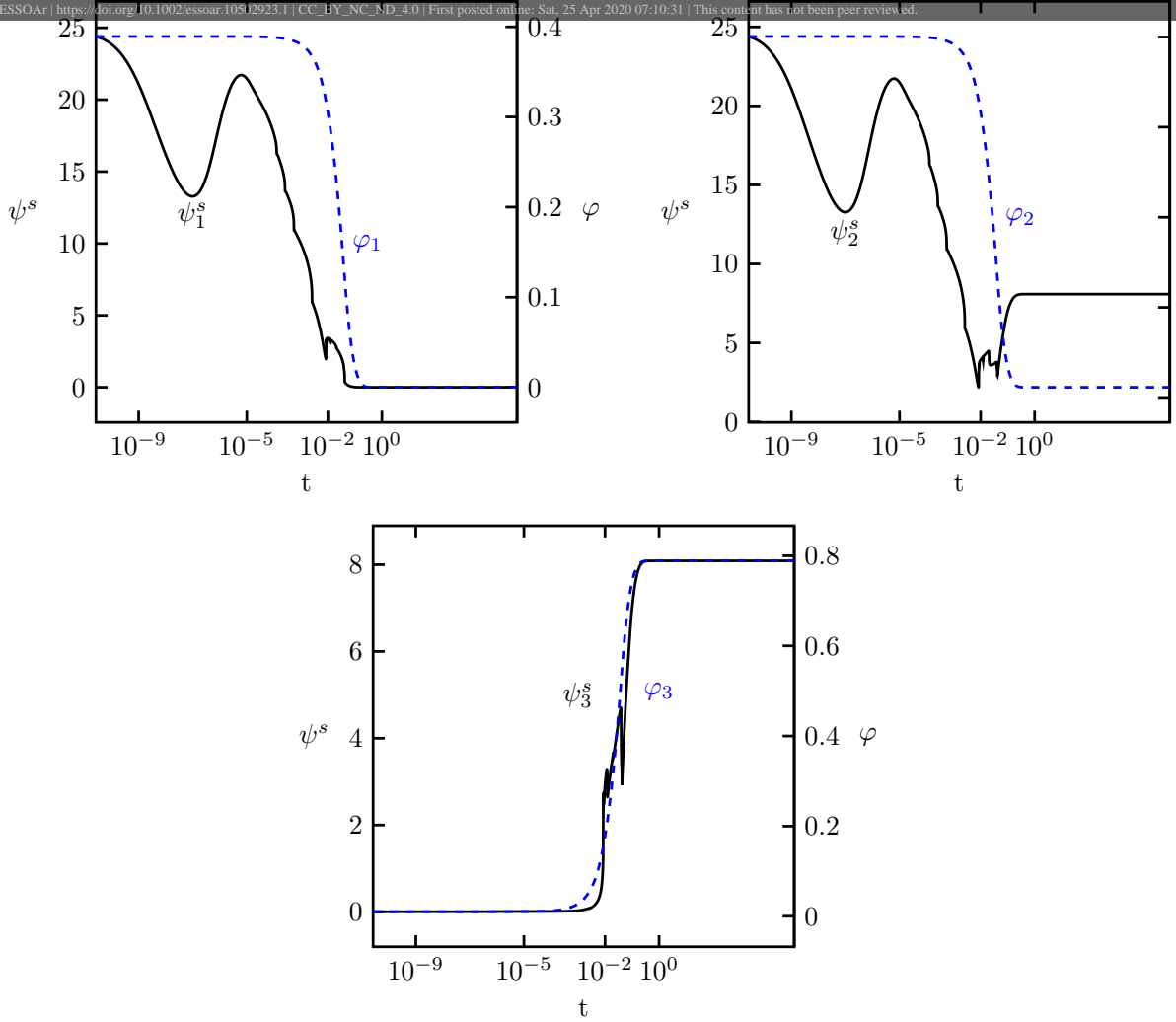
Figure 13.

ESSOAr | https://doi.or

(n) 


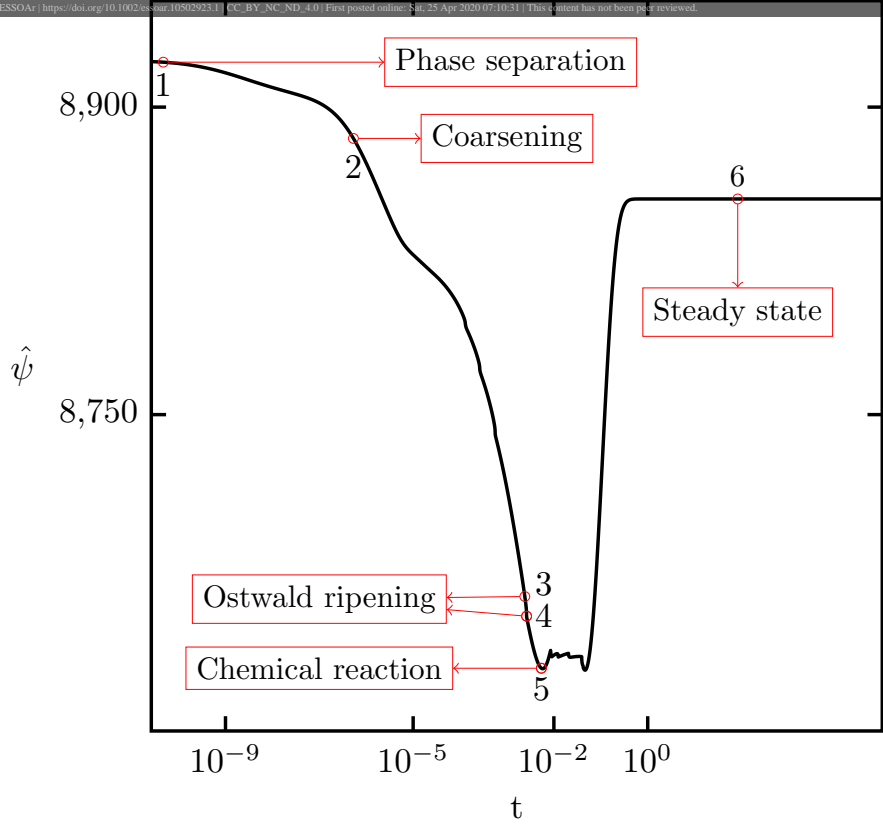


ESSOAr | https://doi.or
Figure 14.

.

(2) 


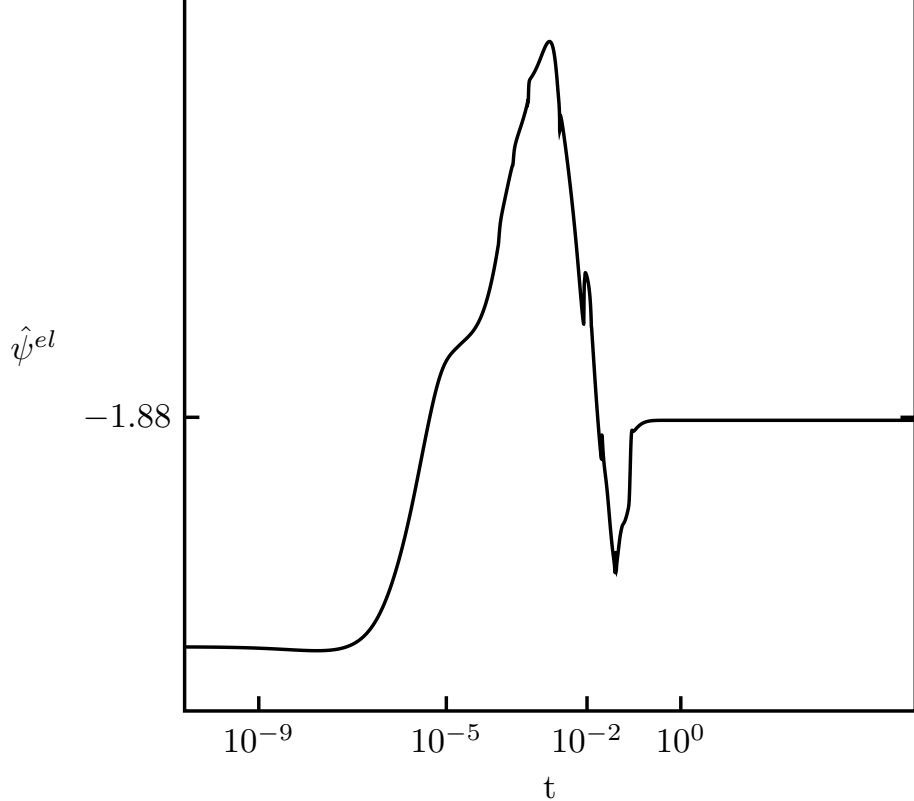

